\title{
Non-Similar Comutational Solutions for Double-Diffusive MHD Transport Phenomena for Non-Newtnian Nanofluid From a Horizontal Circular Cylinder
}

https://doi.org/10.1515/nleng-2018-0035

Received February 15, 2018; revised May 16, 2018; accepted August 3, 2018.

\begin{abstract}
This article aims to study theoretically the combined magneto hydrodynamic flows of casson viscoplastic nanofluid from a horizontal isothermal circular cylinder in non-Darcy porous medium. The impacts of Brownian motion and thermophoresis are consolidated and studied. The governing partial differential equations are converted into nonlinear ordinary differential equations using suitable non-similarity transformation and are solved numerically using Keller-Box finite difference technique. The numerical method is validated with previous published work and the results are found to be in excellent agreement. Numerical results for velocity, temperature, concentration along with skin friction coefficient, heat and mass transfer rate are discussed for various values of physical parameters. It is observed that velocity, heat and mass transfer rate are increased with increasing casson fluid parameter whereas temperature, concentration and skin friction are decreased. Velocity is reduced with increasing Forchheimer parameter whereas temperature and nano-particle concentration are both enhanced. An increase in magnetic parameter is seen to increase temperature and concentration whereas velocity, skin friction heat and mass transfer rate are decreased. The present model finds applications in electric-conductive nano-materials of potential use in aviation and different enterprises, energy systems and thermal enhancement of industrial flow processes.
\end{abstract}

Keywords: Brownian motion; Buoyancy ratio; Buongiorno Nanofluid model; Casson Viscoplastic nanofluid model; Darcy number; Forchheimer parameter; Magnetohydrodynamic; Thermophoresis

\footnotetext{
*Corresponding Author: V. Ramachandra Prasad, Department of Mathematics, School of Advanced Sciences, Vellore Institute of Technology, Vellore - 6322014, Tamil Nadu, India, E-mail: v.ramachandraprasad@vit.ac.in
}

\section{Introduction}

The convection transport study of nanofluid has gained interest in the recent years due to its wide spread application in industry, transportation, electronics, fuel cells, incorporate and medical applications. Usually, base fluids like ethylene glycol, kerosene, water, etc. have low thermal conductivity $[1,2]$. The suspension of nano-sized metallic or non-metallic particles into the base fluids, enhances the thermal conductivity of the base fluids. Choi [3] was the first researcher to use the term Nanofluid, in order to represent the engineered colloids. He observed that on addition of $1 \%$ of nanoparticles to the normal fluid, the fluid thermal conductivity increases twice. Flow, heat and mass transfer of Nanofluids has attracted many researchers due to its prominent role in industry and technology such as electronics, transportation, biomedical (cancer therapy, drug delivery, etc.), micro-electronics, fuel cells, hybridpowered engines, etc. $[1,2]$. The heat transfer enhancement technology has been widely used in refrigerators, automobiles, process and chemical industry, etc. The continuous collision of nanoparticles and molecules of the base fluid is called Brownian motion. The nanoparticle concentration and size of the particle are the most important parameter for enhancing the heat transfer of nanofluid as discussed by Buongiorno [4]. In Buongiorno model [4] the nanoparticle concentration is considered to vary and incorporates the effects of Brownian motion and thermophoresis. Sheikholeslami et al. [5] presented an experimental analysis of hydrothermal behavior of nano-refrigerant duty condensation. Sheikholeslami and Rokni [6] used control volume based finite element method to simulate the effects of Lorentz forces on nanofluid flow

\footnotetext{
S. Abdul gaffar, Department of Mathematics, Salalah College of Technology, Salalah, Oman

B. Rushi Kumar, Department of Mathematics, School of Advanced Sciences, Vellore Institute of Technology, Vellore - 6322014, Tamil Nadu, India
} 
in porous media. Shrivan et al. [7] utilized the finite element method to study the influence of wavy surface characteristics on natural convection flows in a cosine corrugated square cavity filled with Cu-water nanofluid. Rashidi et al. [8] used the volume of fluid model to investigate the nanofluid flow and entropy generation in a single slope solar still using finite volume method. Sheikholeslami and Shehzad [9] considered convective flow of nanofluid inside a porous enclosure by means of two-temperature model taking into account the effects of Lorentz forces. Sheikholeslami [10] numerically investigated the electrohydrodynamic radiative free convection flow of nanofluid in a porous media. Shirvan et al. [11] explored the response surface methodology and two phase mixture model to examine the heat exchanger effectiveness in a double pipe heat exchanger filled with nanofluid. Esfahani et al. [12] used finite volume technique to analyze the entropy generation of nanofluid flow through a wavy channel over heat exchanger plat.

In many fluids the flow properties are difficult to explain by a single constitutive equation like Newtonian model. Geological materials and polymer solutions used in different industries and engineering processes are such fluids which cannot be explained by Newtonian model. The materials that cannot be explained using Newtonian model are called Non-Newtonian fluid models. In past few decades, due to the applications in industries, engineering and technology, non-Newtonian fluid flows has gained interest in researchers $[13,14]$. In such fluids the shear stress and strain rate relation is non-linear. Examples of such fluids include china clay, coal in water, sewage sludge, oil-water emulsions, gas-liquid dispersions, coaloil slurries, detergent and paint production, smart coating and suspension fabrication, pharmacology, cosmetic creams, physiological transport processes (blood, bile and synovial fluid), slurry conveyance, polymer synthesis and food processing. The mathematical models in nonNewtonian fluids are more complicated and relate the shear stresses to the velocity field [15]. Few non-Newtonian transport modeling include oblique micropolar stagnation flows [16], Walter's-B viscoelastic flows [17], Jeffrey's viscoelastic boundary layers [18], magnetized Williamson fluids [19], nanofluid transport from a sphere [20], Maxwell fluids [21], Eyring-Powell fluid [22], Tangent Hyperbolic fluid [23], Oldroyd-B fluid [24] and Power-law model [25]. Of the different non-Newtonian fluids discussed in the literature, the Casson's viscoplastic fluid model [26] is simple and a realistic fluid model that exhibits shear thinning characteristics, yield stress and high shear viscosity [27]. This particular fluid model has infinite viscosity at zero shear rate. The Casson fluid behaves like solid elastic when the shear stress is less than the yield stress and deforms when the shear stress is greater than yield stress. The study of Casson fluid by different researchers include Kumaran and Sandeep [28], Khan et al. [29], Nawaz et al. [30], Kahsif et al. [31], Ahmed et al. [32], Mahanthesh and Gireesh [33] and Rehman et al. [34].

The presence of magnetic field in natural convection flows plays an important role and has many applications like nuclear reactor cooling, magnetohydrodynamic (MHD) generators, geophysics, astrophysics, aerodynamics, plasma engineering, exploration of oil, etc. [35]. Ahmad and Ishak [36] considered an implicit finite difference scheme to study the MHD convection flow of Jeffrey fluid over a stretched sheet immersed in porous medium. Sheikholeslami and Houman [37] investigated the convective heat transfer flow of nanofluid in a porous cavity in the existence of Lorentz forces. Sheikholeslami [38] investigated the MHD forced convection flow of nanofluid inside a porous cavity. He employed CVFEM to simulate the vorticity stream function. Hassan et al. [39] employed homotopy analysis method to examine the nanoparticle shapes behavior on heat and mass transfer flow of ferrofluid over a rotating disk in the presence of low oscillating magnetic field. Ellahi et al. [40] discussed the influence of nanoferroliquid under the low oscillating magnetic field over a stretchable rotating disk. Xie and Jian [41] studied the entropy analysis of magnetohydrodynamic electroosmotic flow. Zeeshan et al. [42] used homotopy analysis method to explore the MHD radiative Couette-Ooiseuille flow of nanofluid in horizontal channel with convective boundary conditions. Recent studies include Abdul Gaffar et al. [4345] and Beg et al. [46].

Flows in fluid saturated porous media has a wide range of applications in different areas of engineering and industry like food processing, fuel cell technologies, geothermics, trickle bed chromatography, etc. Most of studies utilize the Darcy model which is valid for low Reynolds number flows [47]. Srinivasacharya et al. [48] explored the mixed convection flow of viscous fluid past a vertical porous plate with the flow in porous medium is characterized by Darcy-Forchheimer model. Gireesh et al. [49] attempted to analyze the unsteady MHD flow of dusty fluid over stretching surface in non-Darcy porous medium. Natalia et al. [50] presented the mixed convection flow of nanofluid over a vertical flat plate embedded in fluid saturated non-porous medium. Recent studies include Hady et al. [51], V.R. Prasad et al. [52], Som et al. [53], Nazir et al. [54], Abdul gaffar et al. [55, 56].

To the authors' knowledge no studies have been communicated with regard to viscoplastic nanofluid MHD convection flow of isothermal horizontal circular cylinder in 
non-Darcy porous media. The heat transfer with nanoparticles is analyzed by employing Buongiorno model [4]. In the present study a non-similarity mathematical analysis is developed for steady double-diffusive magnetohydrodynamic flows in Casson nanofluid saturated in nonDarcy porous media from a permeable horizontal circular cylinder. The Keller-box difference scheme is used to solve the normalized boundary layer equations and the effects of Forchheimer parameter ( $\Lambda$ ), Brownian motion $(\mathrm{Nb})$, thermophoresis (Nt), buoyancy ratio (Nr) and Darcy number $(D a)$ on the relevant flow variables are described in detail. The present study finds application in solar film collectors, heat exchanger technology, geothermal energy storage systems, etc.

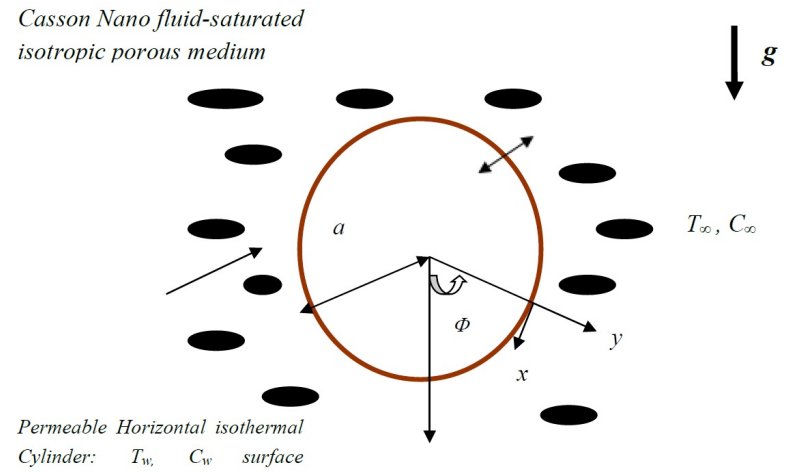

Fig. 1: Physical Model and Coordinate

\section{Viscoplastic Casson Nanofluid Mathematical Model}

The steady-state, laminar, double-diffusive, incompressible, electrically-conducting, MHD convection flows of viscoplastic Nanofluid past a horizontal circular permeable cylinder embedded in fully-saturated porous medium is considered, as shown in Figure 1. An induced magnetic field, $B_{0}$ is assumed to be uniform and acts normal to the surface of the cylinder. A non-Darcy drag force model is employed to simulate porous media and also inertial effects. The $x-\& y$ - coordinates are measured along the circumference and normal to the surface of the cylinder respectively, with $\boldsymbol{a}$ denoting the radius of the cylinder. $\Phi=x / a$ represent the angle of the $y-$ axis with respect to the vertical $0 \leq \Phi \leq \pi$. The gravitational acceleration $\mathbf{g}$, acts downwards. We assume that the Oberbeck-Boussineq approximation holds. Let $T_{w}$ and $C_{w}$ be the constant tem- perature of the cylinder and viscoplastic nanofluid and $T_{\infty}$ and $C_{\infty}$ be the ambient temperature and concentration of the fluid. The governing boundary layer equations in line with Casson [26] \& Buongiorno [4] are:

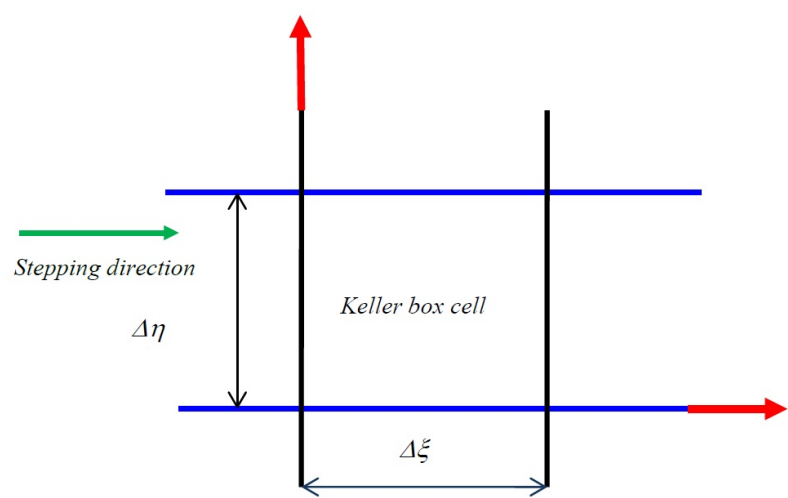

Fig. 2: Keller box computational cell

$$
\begin{gathered}
\frac{\partial u}{\partial x}+\frac{\partial v}{\partial y}=0 \\
u \frac{\partial u}{\partial x}+v \frac{\partial u}{\partial y}=v\left(1+\frac{1}{\beta}\right) \frac{\partial^{2} u}{\partial y^{2}}+g\left[\left(1-C_{\infty}\right) \rho_{f \infty} \beta_{1}\left(T-T_{\infty}\right)\right. \\
\left.-\left(\rho_{p}-\rho_{f \infty}\right)\left(C-C_{\infty}\right)\right] \sin (x / a)-\frac{v}{K} u-\Gamma u^{2}-\frac{\sigma B_{0}^{2}}{\rho} u . \\
u \frac{\partial T}{\partial x}+v \frac{\partial T}{\partial y}=\alpha_{m} \frac{\partial^{2} T}{\partial y^{2}}+\tau\left[D_{B} \frac{\partial C}{\partial y} \frac{\partial T}{\partial y}+\left(\frac{D_{T}}{T_{\infty}}\right)\left(\frac{\partial T}{\partial y}\right)^{2}\right] \\
u \frac{\partial C}{\partial x}+v \frac{\partial C}{\partial y}=D_{B} \frac{\partial^{2} C}{\partial y^{2}}+\left(\frac{D_{T}}{T_{\infty}}\right) \frac{\partial^{2} T}{\partial y^{2}} .
\end{gathered}
$$

Where $\alpha_{m}=\frac{k_{m}}{(\rho c)_{f}} \quad$ and $\quad \tau=\frac{(\rho c)_{p}}{(\rho c)_{f}}$. Where $\rho_{p}$ is the density of the particle, and is the effective heat capacity. The boundary conditions are defined as:

$$
\begin{array}{llll}
\text { At } y=0, & u=v=0, & T=T_{w}, & C=C_{w} \\
\text { As } y \rightarrow \infty, & u=0, & T \rightarrow T_{\infty}, & C \rightarrow C_{\infty} .
\end{array}
$$

Defining the stream function, $\psi$ as $u=\frac{\partial(\psi)}{\partial y}, \quad v=$ $-\frac{\partial(\psi)}{\partial x}$, Eq. (1) is satisfied. We now introduce the following 
dimensionless variables:

$$
\begin{aligned}
& \xi=\frac{x}{a}, \quad \eta=\frac{y}{a} \sqrt[4]{G r}, \quad \psi(\xi, \eta)=v \sqrt[4]{G r} \xi f \\
& \theta(\xi, \eta)=\frac{T-T_{\infty}}{T_{w}-T_{\infty}}, \quad \phi(\xi, \eta)=\frac{C-C_{\infty}}{C_{w}-C_{\infty}} \\
& G r=\frac{\left(1-C_{\infty}\right) \rho_{f \infty} g \beta\left(T_{w}-T_{\infty}\right) a^{3}}{v^{2}}, \quad \operatorname{Pr}=\frac{v}{\alpha}, \\
& L e=\frac{v}{D_{m}}, \quad D a=\frac{K \sqrt{G r}}{a^{2}}, \quad \Lambda=\Gamma a, M=\frac{\sigma B_{0}^{2} a^{2}}{v \rho \sqrt{G r}}, \\
& N b=\frac{\tau D_{B}\left(C_{w}-C_{\infty}\right)}{v}, \quad N t=\frac{\tau D_{T}\left(T_{w}-T_{\infty}\right)}{v T_{\infty}}
\end{aligned}
$$

Using Eq. (6) into Eqs. (2 - 4), reduce as follows:

$$
\begin{gathered}
\left(1+\frac{1}{\beta}\right) f^{\prime \prime \prime}+f^{\prime \prime}-(1+\Lambda \xi) f^{\prime 2}+\frac{\sin \xi}{\xi}(\theta-N r \phi) \\
-\left(\frac{1}{D a}+M\right) f^{\prime}=\xi\left(f^{\prime} \frac{\partial f^{\prime}}{\partial \xi}-f^{\prime \prime} \frac{\partial f}{\partial \xi}\right) . \\
\frac{\theta^{\prime \prime}}{P r}+f \theta^{\prime}+N b \theta^{\prime} \phi^{\prime}+N t \theta^{\prime 2}=\xi\left(f^{\prime} \frac{\partial \theta}{\partial \xi}-\theta^{\prime} \frac{\partial f}{\partial \xi}\right) . \\
\frac{\phi^{\prime \prime}}{L e}+f \phi^{\prime}+\frac{1}{L e} \frac{N t}{N b} \theta^{\prime \prime}=\xi\left(f^{\prime} \frac{\partial \phi}{\partial \xi}-\phi^{\prime} \frac{\partial f}{\partial \xi}\right) .
\end{gathered}
$$

And the transformed non-dimensional boundary conditions are:

At $\eta=0, \quad f^{\prime}=0, \quad f=0, \quad \theta=1, \quad \phi=1$

As $\eta \rightarrow \infty, \quad f^{\prime} \rightarrow 0, \quad \theta \rightarrow 0, \quad \phi \rightarrow 0$

Here primes denote the differentiation with respect to $\eta$. The skin-friction coefficient, Nusselt number and Sherwood number, which are given by:

$$
\begin{gathered}
\frac{1}{2} C_{f} G r^{-3 / 4}=\left(1+\frac{1}{\beta}\right) \xi f^{\prime \prime}(\xi, 0) . \\
\frac{N u}{\sqrt[4]{G r}}=-\theta^{\prime}(\xi, 0) . \\
\frac{L e}{\sqrt[4]{G r}}=-\phi^{\prime}(\xi, 0) .
\end{gathered}
$$

\section{Computational Solution With Keller Box Method (KBM)}

The Keller-Box method (KBM), an implicit difference method is implemented to solve the non-linear boundary layer eqns. (7) - (9) subject to the boundary conditions (10). This technique has remained extremely popular and maintained comparable efficiency to other numerical methods such as finite element, boundary elements, spectral methods etc. the disadvantage of the method however is that the computational effort per time step is expensive due to its step which has to replace the higher derivative by first derivatives, so that the second-order equations can be written as a system of two first-order equations. Also laborious algebraic expressions must be generated for the discretized equations. However KBM has a second order accuracy with arbitrary spacing and attractive extrapolation features. It is unconditionally stable and achieves exceptional accuracy. It converges quickly and provides stable numerical meshing features. KBM provides an improvement in accuracy on explicit or semiimplicit schemes and utilizes customizable stepping in a fully implicit approach. Relevant details are provided in Keller [57]. KBM has been employed extensively in computational non-Newtonian transport modelling. The KellerBox discretization is fully coupled at each step which reflects the physics of parabolic systems - which are also fully coupled. Discrete calculus associated with the KellerBox scheme has also been shown to be fundamentally different from all other mimetic (physics capturing) numerical methods. The Keller Box Scheme comprises four stages.

1. Decomposition of the $N^{\text {th }}$ order partial differential equation system to $N$ first order equations.

2. Finite Difference Discretization

3. Quasilinearization of Non-Linear Keller Algebraic Equations and finally.

4. Block-tridiagonal Elimination solution of the Linearized Keller Algebraic Equations.

\section{Step1: Reduction of the $\mathbf{N}^{\text {th }}$ order partial differential equation system to $\boldsymbol{N}$ first order equations}

New variables are introduced to Eqns. (7) - (9) and (10), to render the boundary value problem as a multiple system of first order equations. A set of eight simultaneous first order differential equations are therefore generated by introducing the new variables:

$$
\begin{aligned}
& u(x, y)=f^{\prime}, v(x, y)=f^{\prime \prime}, g(x, y)=\phi, g^{\prime}(x, y)=p, \\
& s(x, y)=\theta, t(x, y)=\theta^{\prime} .
\end{aligned}
$$

$$
\begin{aligned}
& f^{\prime}=u . \\
& u^{\prime}=v . \\
& g^{\prime}=p . \\
& s^{\prime}=t .
\end{aligned}
$$




$$
\begin{aligned}
\left(1+\frac{1}{\beta}\right) v^{\prime}+f v-(1+\Lambda \xi) u^{2}-\left(\frac{1}{D a}+M\right) u+B(s-N r g) & \\
=\xi\left[u \frac{\partial u}{\partial \xi}-v \frac{\partial f}{\partial \xi}\right] & \\
\frac{t^{\prime}}{\operatorname{Pr}}+f t+N b t p+N t t^{2} & =\xi\left(u \frac{\partial s}{\partial \xi}-t \frac{\partial f}{\partial \xi}\right) \\
\frac{p^{\prime}}{L e}+f p+\frac{1}{L e} \frac{N t}{N b} t^{\prime} & =\xi\left(u \frac{\partial g}{\partial \xi}-p \frac{\partial f}{\partial \xi}\right)
\end{aligned}
$$

where primes denote differentiation with respect to $\eta$. In terms of the dependent variables, the boundary conditions become:

$$
\begin{array}{llll}
\text { At } & \eta=0, \quad f=0, \quad u=0, & s=1, & g=1 \\
\text { As } \quad \eta \rightarrow \infty, \quad u \rightarrow 0, \quad s \rightarrow 0, & g \rightarrow 0 .
\end{array}
$$

\section{Step 2: Finite Difference Discretization}

A two-dimensional computational grid is imposed on the $\xi-\eta$ plane as sketched in Fig. 2 The stepping process is defined by:

$$
\begin{gathered}
\eta_{0}=0, \quad \eta_{j}=\eta_{j-1}+h_{j}, \quad j=1,2, \ldots, J, \quad \eta_{J} \equiv \eta_{\infty} \\
\xi^{0}=0, \quad \xi^{n}=\xi^{n-1}+k_{n}, \quad n=1,2, \ldots, N
\end{gathered}
$$

where $k_{n}$ is the $\Delta \xi$ - spacing and $h_{j}$ is the $\Delta \eta$ - spacing. If $g_{j}^{n}$ denotes the value of any variable at $\left(\eta_{j}, \xi^{n}\right)$, then the variables and derivatives of Equations (15) - (21) at $\left(\eta_{j-1 / 2}, \xi^{n-1 / 2}\right)$ are replaced by:

$$
\begin{gathered}
g_{j-1 / 2}^{n-1 / 2}=\frac{1}{4}\left(g_{j}^{n}+g_{j-1}^{n}+g_{j}^{n-1}+g_{j-1}^{n-1}\right) . \\
\left(\frac{\partial g}{\partial \eta}\right)_{j-1 / 2}^{n-1 / 2}=\frac{1}{2 h_{j}}\left(g_{j}^{n}-g_{j-1}^{n}+g_{j}^{n-1}-g_{j-1}^{n-1}\right) . \\
\left(\frac{\partial g}{\partial \xi}\right)_{j-1 / 2}^{n-1 / 2}=\frac{1}{2 k^{n}}\left(g_{j}^{n}-g_{j-1}^{n}+g_{j}^{n-1}-g_{j-1}^{n-1}\right) .
\end{gathered}
$$

The resulting finite - difference approximation of equations (15) - (21) for the mid - point $\left(\eta_{j-1 / 2}, \xi^{n}\right)$, are:

$$
\begin{aligned}
& h_{j}^{-1}\left(f_{j}^{n}-f_{j-1}^{n}\right)=u_{j-1 / 2}^{n} . \\
& h_{j}^{-1}\left(u_{j}^{n}-u_{j-1}^{n}\right)=v_{j-1 / 2}^{n} . \\
& h_{j}^{-1}\left(v_{j}^{n}-v_{j-1}^{n}\right)=q_{j-1 / 2}^{n} . \\
& h_{j}^{-1}\left(g_{j}^{n}-g_{j-1}^{n}\right)=p_{j-1 / 2}^{n} .
\end{aligned}
$$

where we have used the abbreviations

$$
\alpha=\frac{\xi^{n-1 / 2}}{k_{n}}, \quad B=\frac{\sin \left(\xi^{n-1 / 2}\right)}{\xi^{n-1 / 2}}
$$

$$
\begin{aligned}
& \left(1+\frac{1}{\beta}\right)\left(v_{j}-v_{j-1}\right)+\frac{h_{j}(1+\alpha)}{4}\left(f_{j}+f_{j-1}\right)\left(v_{j}+v_{j-1}\right) \\
& -(1+\alpha+\Lambda \xi) \frac{h_{j}}{16}\left(u_{j}+u_{j-1}\right)^{2}-\left(\frac{1}{D a}+M\right) \frac{h_{j}}{2}\left(u_{j}+u_{j-1}\right) \\
& +\frac{B h_{j}}{2}\left(s_{j}+s_{j-1}-N r\left(g_{j}+g_{j-1}\right)\right)-\frac{\alpha h_{j}}{2} f_{j-1 / 2}^{n-1}\left(v_{j}+v_{j-1}\right) \\
& +\frac{\alpha h_{j}}{2} v_{j-1 / 2}^{n-1}\left(f_{j}+f_{j-1}\right)=\left[R_{1}\right]_{j-/ 12}^{n-1} . \\
& \quad \frac{1}{\operatorname{Pr}}\left(t_{j}-t_{j-1}\right)+\frac{(1+\alpha) h_{j}}{4}\left(f_{j}+f_{j-1}\right)\left(t_{j}+t_{j-1}\right) \\
& \quad+\frac{N b h_{j}}{4}\left(t_{j}+t_{j-1}\right)\left(p_{j}+p_{j-1}\right)+\frac{N t h_{j}}{4}\left(t_{j}+t_{j-1}\right)^{2} \\
& \quad-\frac{\alpha h_{j}}{4}\left(u_{j}+u_{j-1}\right)\left(s_{j}+s_{j-1}\right)+\frac{\alpha h_{j}}{2} s_{j-1 / 2}^{n-1}\left(u_{j}+u_{j-1}\right) \\
& \quad-\frac{\alpha h_{j}}{2} u_{j-1 / 2}^{n-1}\left(s_{j}+s_{j-1}\right)-\frac{\alpha h_{j}}{2} f_{j-1 / 2}^{n-1}\left(t_{j}+t_{j-1}\right) \\
& \quad+\frac{\alpha h_{j}}{2} t_{j-1 / 2}^{n-1}\left(f_{j}+f_{j-1}\right)=\left[R_{2}\right]_{j-1 / 2}^{n-1} .
\end{aligned}
$$

$$
\begin{aligned}
& \frac{1}{L e}\left(p_{j}-p_{j-1}\right)+\frac{(1+\alpha) h_{j}}{4}\left(f_{j}+f_{j-1}\right)\left(p_{j}+p_{j-1}\right) \\
& +\frac{1}{L e} \frac{N t}{N b}\left(t_{j}-t_{j-1}\right)-\frac{\alpha h_{j}}{4}\left(u_{j}+u_{j-1}\right)\left(g_{j}+g_{j-1}\right) \\
& +\frac{\alpha h_{j}}{2} g_{j-1 / 2}^{n-1}\left(u_{j}+u_{j-1}\right)-\frac{\alpha h_{j}}{2} u_{j-1 / 2}^{n-1}\left(g_{j}+g_{j-1}\right) \\
& -\frac{\alpha h_{j}}{2} f_{j-1 / 2}^{n-1}\left(p_{j}+p_{j-1}\right)+\frac{\alpha h_{j}}{2} p_{j-1 / 2}^{n-1}\left(f_{j}+f_{j-1}\right)=\left[R_{3}\right]_{j-1 / 2}^{n-1} .
\end{aligned}
$$

$\left[R_{1}\right]_{j-1 / 2}^{n-1}=$

$(27)-h_{j}\left[\begin{array}{c}\left(1+\frac{1}{\beta}\right)\left(v^{\prime}\right)_{j-1 / 2}^{n-1}+(1-\alpha)(f v)_{j-1 / 2}^{n-1}-(1-\alpha-\Lambda \xi)\left(u_{j-1}^{n-1}\right)^{2} \\ -\left(\frac{1}{D a}+M\right) u_{j-1}^{n-1}+B\left(s_{j-1 / 2}^{n-1}-N r g_{j-1 / 2}^{n-1}\right)\end{array}\right]$.

$$
\begin{aligned}
& {\left[R_{2}\right]_{j-1 / 2}^{n-1}=-h_{j}\left[\frac{1}{P r}\left(t^{\prime}\right)_{j-1 / 2}^{n-1}+(1-\alpha)(f t)_{j-1 / 2}^{n-1}\right.} \\
& \left.+N b(t p)_{j-1 / 2}^{n-1}+N t\left(t^{2}\right)_{j-1 / 2}^{n-1}+\alpha(u s)_{j-1 / 2}^{n-1}\right] . \\
& {\left[R_{3}\right]_{j-1 / 2}^{n-1}=-h_{j}\left[\frac{1}{L e}\left(p^{\prime}\right)_{j-1 / 2}^{n-1}+(1-\alpha)(f p)_{j-1 / 2}^{n-1}\right.} \\
& \left.+\frac{1}{L e} \frac{N t}{N b}\left(t^{\prime}\right)_{j-1 / 2}^{n-1}+\alpha(u g)_{j-1 / 2}^{n-1}\right] .
\end{aligned}
$$


The boundary conditions are:

$$
f_{0}^{n}=u_{0}^{n}=0, s_{0}^{n}=1, g_{0}^{n}=1, u_{J}^{n}=0, s_{J}^{n}=0, g_{J}^{n}=0 .
$$

Stage 3: Quasilinearization of Non-Linear Keller Algebraic Equations

If we assume $f_{j}^{n-1}, u_{j}^{n-1}, v_{j}^{n-1}, g_{j}^{n-1}, p_{j}^{n-1}, s_{j}^{n-1}, t_{j}^{n-1}$ to be known for $0 \leq j \leq J$, this leads to a system of $8 J+8$ equations for the solution of $8 J+8$ unknowns $f_{j}^{n}, u_{j}^{n}, v_{j}^{n}, g_{j}^{n}, p_{j}^{n}, s_{j}^{n}, t_{j}^{n}, j=0,1,2, \ldots, J$. This non-linear system of algebraic equations is linearized by means of Newton's method.

\section{Stage 4: Block-tridiagonal Elimination Solution of Lin- ear Keller Algebraic Equations}

The linearized system is solved by the blockelimination method, since it possess a block-tridiagonal structure. The bock-tridiagonal structure generated consists of block matrices. The complete linearized system is formulated as a block matrix system, where each element in the coefficient matrix is a matrix itself, and this system is solved using the efficient Keller-box method. The numerical results are strongly influenced by the number of mesh points in both directions. After some trials in the $\eta$-direction (radial coordinate) a larger number of mesh points are selected whereas in the $\xi$-direction (tangential coordinate) significantly less mesh points are utilized. $\eta_{\max }$ has been set at 16.0 and this defines an adequately large value at which the prescribed boundary conditions are satisfied. $\xi_{\max }$ is set at 3.0 for this flow domain. Mesh independence is achieved in the present computations. The numerical algorithm is executed in MATLAB on a PC. The method demonstrates excellent stability, convergence and consistency, as elaborated by Keller [57].

\section{Interpretation of Results}

The system of Eqs. (7 - 9) subject to Eq. (10) are numerically solved using an implicit finite difference Keller-Box technique and is programmed using MATLAB. The accuracy present code is validated and presented in Table 1 by comparing the present results of heat transfer rate with those of Merkin [58] and Yih [59] for different values of $\xi$. It has been found that the present results are in good correlation. In Tables 2 and 3 we present the effects of $\beta, \Lambda$ and $\operatorname{Pr}$ on $C_{f}, N u$ and $S h$ along with a variation in $\xi$. With increasing $\beta$, the $C_{f}$ is reduced whereas $N u$ is enhanced. A small increment in $S h$ is observed with increasing $\beta$. An increasing $P r$ is found to increase $C_{f}$ slightly whereas $\mathrm{Nu}$ is reduced. However, $S h$ is strongly increased with $P r$. With increasing $\Lambda$, a significant decrease in $C_{f}, N u$ and $S h$ is ob- served. This trend is sustained for $\xi>0$. Tables 4 and 5 present the impact of $\mathrm{Da}$ and $\mathrm{Le}$ on $C_{f}, \mathrm{Nu}$ and Sh along with a variation in $\xi$. An increasing $D a$ is seen to increase $C_{f}$ significantly. Also an increase in $D a$ is observed to increase both $\mathrm{Nu}$ and $S h$. An increasing $D a$ is seen to increase $C_{f}$ significantly. In these Tables we also present the results of $C_{f}, N u$ and $S h$ for different values of $L e$. Also an increasing $L e$ is observed to increase $C_{f}$ significantly. Whereas, $N u$ is reduced with increasing $L e$ values. The $S h$ is strongly increased with increasing $L e$. This trend is sustained for $\xi>$ 0 .

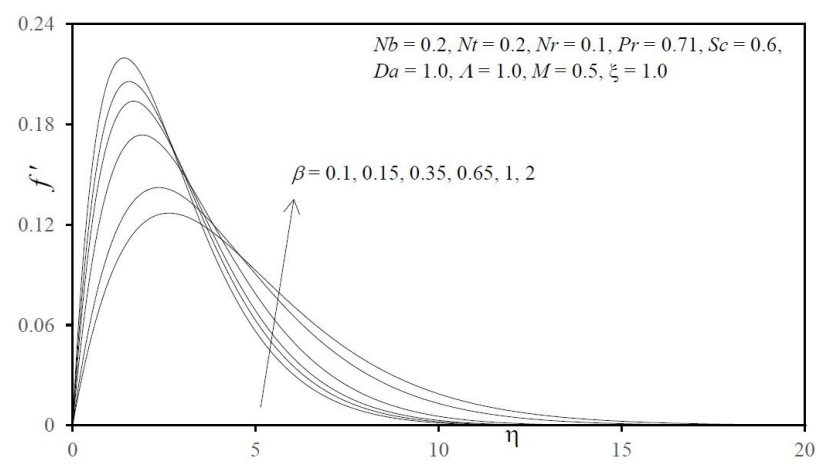

(a)

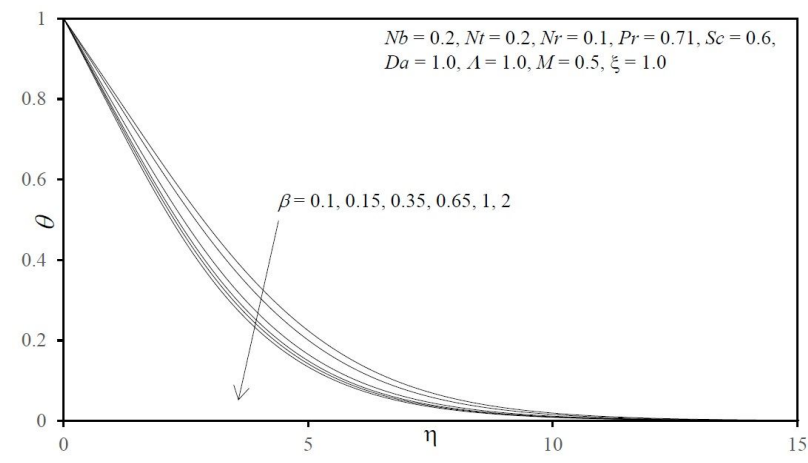

(b)

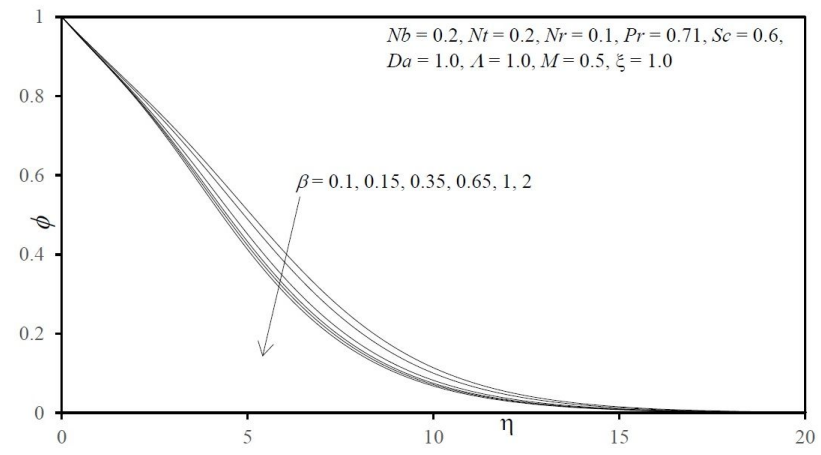

(c)

Fig. 3: (a) Influence of $\beta$ on Velocity Profiles; (b) Influence of $\beta$ on Temperature Profiles; (c) Influence of $\beta$ on Concentration Profiles 
Table 1: The values of the Local heat transfer coefficient $(N u)$ for different values of $\xi$ with $\operatorname{Pr}=0.71, \beta \rightarrow \infty, \Lambda=0, D a \rightarrow \infty$ and $N b=N r=$ $N t=0, L e=0.6, M=0.5$

\begin{tabular}{cccc}
\hline$\xi$ & \multicolumn{3}{c}{$N u \mathrm{Gr}^{-1 / 4}=-\theta^{\prime}(\xi, 0)$} \\
\cline { 2 - 4 } & Merkin [58] & Yih [59] & Present \\
\hline 0.0 & 0.4212 & 0.4214 & 0.4211 \\
0.2 & 0.4204 & 0.4207 & 0.4206 \\
0.4 & 0.4182 & 0.4184 & 0.4185 \\
0.6 & 0.4145 & 0.4147 & 0.4146 \\
0.8 & 0.4093 & 0.4096 & 0.4095 \\
1.0 & 0.4025 & 0.4030 & 0.4027 \\
1.2 & 0.3942 & 0.3950 & 0.3947 \\
1.4 & 0.3843 & 0.3854 & 0.3852 \\
1.6 & 0.3727 & 0.3740 & 0.3735 \\
1.8 & 0.3594 & 0.3608 & 0.3598 \\
2.0 & 0.3443 & 0.3457 & 0.3448 \\
2.2 & 0.3270 & 0.3283 & 0.3280 \\
2.4 & 0.3073 & 0.3086 & 0.3076 \\
2.6 & 0.2847 & 0.2860 & 0.2852 \\
2.8 & 0.2581 & 0.2595 & 0.2592 \\
3.0 & 0.2252 & 0.2267 & 0.2255 \\
$\pi$ & 0.1963 & 0.1962 & 0.1961 \\
\hline
\end{tabular}

Table 2: Values of $C_{f}, N u$ and $S h$ for various values of $\beta, \operatorname{Pr}, S c$ and $\xi(N b=N t=0.2, N r=0.1, D a=1.0, M=0.5, L e=0.6)$

\begin{tabular}{|c|c|c|c|c|c|c|c|c|c|c|c|}
\hline \multirow{2}{*}{$\beta$} & \multirow{2}{*}{$\Lambda$} & \multirow{2}{*}{$\operatorname{Pr}$} & \multicolumn{3}{|c|}{$\xi=0^{0}$} & \multicolumn{3}{|c|}{$\xi=30^{\circ}$} & \multicolumn{3}{|c|}{$\xi=60^{0}$} \\
\hline & & & $C_{f}$ & $\mathrm{Nu}$ & Sh & $C_{f}$ & $\mathrm{Nu}$ & Sh & $C_{f}$ & $\mathrm{Nu}$ & $S h$ \\
\hline 0.1 & \multirow{5}{*}{1.0} & \multirow{11}{*}{0.71} & 0 & 0.1791 & 0.1012 & 0.6901 & 0.1745 & 0.0981 & 1.2171 & 0.1638 & 0.0912 \\
\hline 0.15 & & & 0 & 0.1902 & 0.1042 & 0.6067 & 0.1850 & 0.1008 & 1.0678 & 0.1732 & 0.0934 \\
\hline 0.35 & & & 0 & 0.2111 & 0.1086 & 0.4699 & 0.2046 & 0.1048 & 0.8236 & 0.1907 & 0.0961 \\
\hline 0.65 & & & 0 & 0.2235 & 0.1103 & 0.3995 & 0.2161 & 0.1058 & 0.6383 & 0.2007 & 0.0970 \\
\hline 1.0 & & & 0 & 0.2303 & 0.1110 & 0.3634 & 0.2223 & 0.1063 & 0.6341 & 0.2061 & 0.0973 \\
\hline \multirow[t]{6}{*}{2.0} & & & 0 & 0.2383 & 0.1114 & 0.3234 & 0.2295 & 0.1066 & 0.5632 & 0.2123 & 0.0974 \\
\hline & 0.01 & & 0 & 0.2303 & 0.1109 & 0.3682 & 0.2253 & 0.1081 & 0.6497 & 0.2113 & 0.1004 \\
\hline & 5 & & 0 & 0.2303 & 0.1109 & 0.3473 & 0.2122 & 0.1002 & 0.5880 & 0.1905 & 0.0886 \\
\hline & 10 & & 0 & 0.2303 & 0.1109 & 0.3322 & 0.2024 & 0.0947 & 0.5506 & 0.1775 & 0.0820 \\
\hline & 15 & & 0 & 0.2303 & 0.1109 & 0.3207 & 0.1948 & 0.0907 & 0.5242 & 0.1683 & 0.0778 \\
\hline & 20 & & 0 & 0.2303 & 0.1109 & 0.3116 & 0.1887 & 0.0876 & 0.5041 & 0.1612 & 0.0748 \\
\hline \multirow{7}{*}{1.0} & 30 & & 0 & 0.2303 & 0.1109 & 0.2980 & 0.1795 & 0.0834 & 0.4749 & 0.1509 & 0.0708 \\
\hline & \multirow{6}{*}{1.0} & 0.5 & 0 & 0.3246 & 0.3722 & 0.3156 & 0.3214 & 0.3711 & 0.5458 & 0.3141 & 0.3683 \\
\hline & & 1 & 0 & 0.3157 & 0.3808 & 0.3159 & 0.3105 & 0.3816 & 0.5491 & 0.2986 & 0.3834 \\
\hline & & 2 & 0 & 0.2976 & 0.3981 & 0.3163 & 0.2904 & 0.4009 & 0.5534 & 0.2735 & 0.4075 \\
\hline & & 3 & 0 & 0.2795 & 0.4153 & 0.3167 & 0.2715 & 0.4193 & 0.5561 & 0.2526 & 0.4282 \\
\hline & & 5 & 0 & 0.2448 & 0.4488 & 0.3176 & 0.2364 & 0.4532 & 0.5600 & 0.2168 & 0.4631 \\
\hline & & 7 & 0 & 0.2128 & 0.4796 & 0.3187 & 0.2048 & 0.4838 & 0.5632 & 0.1862 & 0.4930 \\
\hline
\end{tabular}

Figs. 3 - 12 illustrate the profiles of velocity and temperature for different values of the thermophysical parameters, viz., $\beta, N b, N t, N r, L e, D a, \Lambda, \operatorname{Pr}$ and $M$. The default values of for these parameters are: $\beta=D a=\Lambda=1.0, \operatorname{Pr}=$ $0.71, N b=N t=0.2, N r=0.1, L e=0.6, M=0.5$ and $\xi=1.0$.

Figs. 3(a) - 3(c) present the impact of $\beta$ on velocity $\left(f^{\prime}\right)$, temperature $(\theta)$ and concentration $(\varphi)$. It is observed that increasing $\beta$ values increases velocity near the cylinder surface but decreases it further away i.e., the viscoplastic fluid behaves as Newtonian fluid as $\beta$ increases. It is ob- served that an increase in $\beta$ decreases the yield stress and hence decreases the momentum boundary layer thickness. This contraction in boundary layer thickness is caused due to the tensile stress. Whereas, the temperature and spices concentration are decreased slightly throughout the boundary layer regime. The viscoplastic fluid parameter $\beta$ appears only in the momentum boundary layer Eq. (7) via the shear term $(1+1 / \beta) f^{\prime \prime \prime}$. The momentum equation couples strongly with the energy and species concentration equations via the thermal and species buoyancy force 
Table 3: Values of $C_{f}$, Nu and $S h$ for various values of $\beta, P r, S c$ and $\xi(N b=N t=0.2, N r=0.1, D a=1.0, M=0.5, L e=0.6)$

\begin{tabular}{|c|c|c|c|c|c|c|c|c|c|c|c|}
\hline \multirow{2}{*}{$\beta$} & \multirow{2}{*}{$\Lambda$} & \multirow{2}{*}{$\operatorname{Pr}$} & \multicolumn{3}{|c|}{$\xi=90^{\circ}$} & \multicolumn{3}{|c|}{$\xi=120^{\circ}$} & \multicolumn{3}{|c|}{$\xi=180^{\circ}$} \\
\hline & & & $C_{f}$ & $\mathrm{Nu}$ & Sh & $C_{f}$ & $\mathrm{Nu}$ & Sh & $C_{f}$ & $\mathrm{Nu}$ & Sh \\
\hline 0.1 & \multirow{5}{*}{1.0} & & 1.4624 & 0.1464 & 0.0804 & 1.3511 & 0.1213 & 0.0652 & 0.1059 & 0.0246 & 0.0074 \\
\hline 0.15 & & & 1.2793 & 0.1544 & 0.0819 & 1.1771 & 0.1273 & 0.0653 & 0.0898 & 0.0249 & 0.0078 \\
\hline 0.35 & & & 0.9813 & 0.1689 & 0.0835 & 0.8957 & 0.1378 & 0.0657 & 0.0645 & 0.0250 & 0.0083 \\
\hline 0.65 & & & 0.8292 & 0.1770 & 0.0838 & 0.7534 & 0.1435 & 0.0658 & 0.0533 & 0.0252 & 0.0090 \\
\hline 1.0 & & & 0.7516 & 0.1813 & 0.0839 & 0.6812 & 0.1465 & 0.0659 & 0.0474 & 0.0254 & 0.0102 \\
\hline \multirow[t]{6}{*}{2.0} & & 0.71 & 0.6661 & 0.1892 & 0.0840 & 0.6020 & 0.1498 & 0.0661 & 0.0413 & 0.0255 & 0.0108 \\
\hline & 0.01 & 0.71 & 0.7771 & 0.1878 & 0.0875 & 0.7087 & 0.1531 & 0.0689 & 0.0482 & 0.0258 & 0.0093 \\
\hline & 5 & & 0.6831 & 0.1635 & 0.0748 & 0.6128 & 0.1299 & 0.0582 & 0.0452 & 0.0218 & 0.0085 \\
\hline & 10 & & 0.6325 & 0.1503 & 0.0689 & 0.5651 & 0.1184 & 0.0542 & 0.0433 & 0.0201 & 0.0081 \\
\hline & 15 & & 0.5985 & 0.1414 & 0.0656 & 0.5336 & 0.1110 & 0.0521 & 0.0418 & 0.0189 & 0.0077 \\
\hline & 20 & & 0.5731 & 0.1348 & 0.0633 & 0.5103 & 0.1056 & 0.0509 & 0.0405 & 0.0181 & 0.0075 \\
\hline \multirow{7}{*}{1.0} & 30 & & 0.5367 & 0.1254 & 0.0606 & 0.4772 & 0.0980 & 0.0497 & 0.0381 & 0.0169 & 0.0071 \\
\hline & \multirow{6}{*}{1.0} & 0.5 & 0.6337 & 0.3036 & 0.3646 & 0.5524 & 0.2911 & 0.3608 & 0.0291 & 0.2731 & 0.3570 \\
\hline & & 1 & 0.6429 & 0.2805 & 0.3870 & 0.5676 & 0.2573 & 0.3932 & 0.0310 & 0.2134 & 0.4121 \\
\hline & & 2 & 0.6551 & 0.2470 & 0.4195 & 0.5888 & 0.2103 & 0.4375 & 0.0351 & 0.1101 & 0.5021 \\
\hline & & 3 & 0.6627 & 0.2225 & 0.4432 & 0.6020 & 0.1801 & 0.4656 & 0.0382 & 0.0499 & 0.5471 \\
\hline & & 5 & 0.6721 & 0.1857 & 0.4790 & 0.6174 & 0.1419 & 0.5014 & 0.0407 & 0.0127 & 0.5618 \\
\hline & & 7 & 0.6784 & 0.1578 & 0.5073 & 0.6266 & 0.1161 & 0.5259 & 0.0412 & 0.0061 & 0.5569 \\
\hline
\end{tabular}

Table 4: Values of $C_{f}, N u$ and $S h$ for different $D a$, Le and $\xi(\beta=1.0, N b=N t=0.2, N t=0.1, \Lambda=1.0, M=0.5, P r=0.71)$

\begin{tabular}{|c|c|c|c|c|c|c|c|c|c|c|}
\hline \multirow{2}{*}{$D a$} & \multirow{2}{*}{ Le } & \multicolumn{3}{|c|}{$\xi=0^{0}$} & \multicolumn{3}{|c|}{$\xi=30^{\circ}$} & \multicolumn{3}{|c|}{$\xi=60^{\circ}$} \\
\hline & & $C_{f}$ & $\mathrm{Nu}$ & $S h$ & $C_{f}$ & $\mathrm{Nu}$ & $S h$ & $C_{f}$ & $\mathrm{Nu}$ & $S h$ \\
\hline \multirow{6}{*}{1.0} & 0.25 & 0 & 0.2313 & -0.0192 & 0.3584 & 0.2234 & -0.0171 & 0.6263 & 0.2074 & -0.0129 \\
\hline & 0.75 & 0 & 0.2299 & 0.1543 & 0.3650 & 0.2218 & 0.1481 & 0.6368 & 0.2057 & 0.1362 \\
\hline & 1.0 & 0 & 0.2292 & 0.2133 & 0.3672 & 0.2211 & 0.2053 & 0.6403 & 0.2050 & 0.1895 \\
\hline & 2.0 & 0 & 0.2274 & 0.3683 & 0.3721 & 0.2193 & 0.3558 & 0.6485 & 0.2033 & 0.3308 \\
\hline & 3.0 & 0 & 0.2264 & 0.4692 & 0.3749 & 0.2183 & 0.4540 & 0.6531 & 0.2023 & 0.4230 \\
\hline & 5.0 & 0 & 0.2252 & 0.6101 & 0.3780 & 0.2171 & 0.5912 & 0.6585 & 0.2012 & 0.5522 \\
\hline 0.1 & \multirow{6}{*}{0.6} & 0 & 0.1287 & 0.0713 & 0.1832 & 0.1244 & 0.0699 & 0.3164 & 0.1130 & 0.0662 \\
\hline 0.15 & & 0 & 0.1481 & 0.0738 & 0.2159 & 0.1431 & 0.0719 & 0.3733 & 0.1304 & 0.0671 \\
\hline 0.2 & & 0 & 0.1625 & 0.0771 & 0.2402 & 0.1571 & 0.0749 & 0.4161 & 0.1435 & 0.0692 \\
\hline 0.25 & & 0 & 0.1736 & 0.0807 & 0.2594 & 0.1679 & 0.0781 & 0.4499 & 0.1538 & 0.0718 \\
\hline 0.3 & & 0 & 0.1826 & 0.0841 & 0.2750 & 0.1765 & 0.0813 & 0.4774 & 0.1620 & 0.0745 \\
\hline 0.35 & & 0 & 0.1899 & 0.0873 & 0.2880 & 0.1836 & 0.0842 & 0.5003 & 0.1688 & 0.0771 \\
\hline
\end{tabular}

Table 5: Values of $C_{f}$, Nu and $S h$ for different $D a$, Le and $\xi(\beta=1.0, N b=N t=0.2, N t=0.1, \Lambda=1.0, M=0.5, \operatorname{Pr}=0.71)$

\begin{tabular}{ccccccccccc}
\hline \multirow{2}{*}{ Da } & \multirow{2}{*}{ Le } & \multicolumn{3}{c}{$\xi=90^{0}$} & \multicolumn{3}{c}{$\xi=120^{0}$} & \multicolumn{3}{c}{$\xi=180^{0}$} \\
\cline { 3 - 10 } & & $C_{f}$ & $N u$ & $S h$ & $C_{f}$ & $N u$ & $S h$ & $C_{f}$ & $N u$ & $S h$ \\
\hline & 0.25 & 0.7435 & 0.1826 & -0.0072 & 0.6753 & 0.1477 & -0.0009 & 0.0474 & 0.0248 & 0.0000 \\
& 0.75 & 0.7544 & 0.1809 & 0.1181 & 0.6835 & 0.1461 & 0.0932 & 0.0475 & 0.0245 & 0.0129 \\
1.0 & 1.0 & 0.7583 & 0.1802 & 0.1656 & 0.6865 & 0.1456 & 0.1324 & 0.0475 & 0.0244 & 0.0206 \\
& 2.0 & 0.7475 & 0.1787 & 0.2922 & 0.6942 & 0.1444 & 0.2379 & 0.0477 & 0.0242 & 0.0429 \\
& 3.0 & 0.7727 & 0.1779 & 0.3751 & 0.6986 & 0.1437 & 0.3073 & 0.0478 & 0.0241 & 0.0581 \\
& 5.0 & 0.7789 & 0.1770 & 0.4913 & 0.7040 & 0.1430 & 0.4048 & 0.0480 & 0.0240 & 0.0801 \\
0.1 & & 0.3645 & 0.0948 & 0.0593 & 0.3137 & 0.0791 & 0.0479 & 0.0162 & 0.0059 & 0.0035 \\
0.15 & & 0.4318 & 0.1099 & 0.0602 & 0.3739 & 0.0819 & 0.0484 & 0.0197 & 0.0060 & 0.0035 \\
0.2 & 0.6 & 0.4829 & 0.1217 & 0.0603 & 0.4203 & 0.0916 & 0.0485 & 0.0225 & 0.0072 & 0.0039 \\
0.25 & & 0.5235 & 0.1311 & 0.0620 & 0.4579 & 0.0996 & 0.0494 & 0.0250 & 0.0095 & 0.0049 \\
0.3 & & 0.5569 & 0.1388 & 0.0640 & 0.4892 & 0.1064 & 0.0507 & 0.0272 & 0.0098 & 0.0078 \\
0.35 & & 0.5850 & 0.1452 & 0.0660 & 0.5158 & 0.1121 & 0.0517 & 0.0291 & 0.0112 & 0.0181 \\
\hline
\end{tabular}




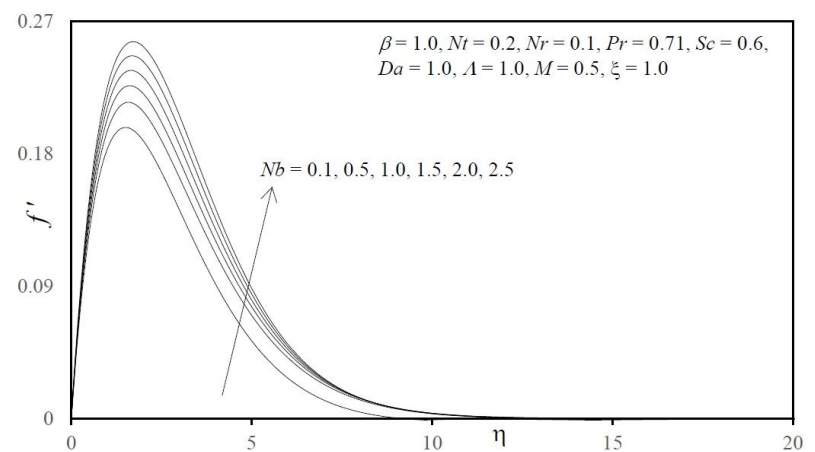

(a)

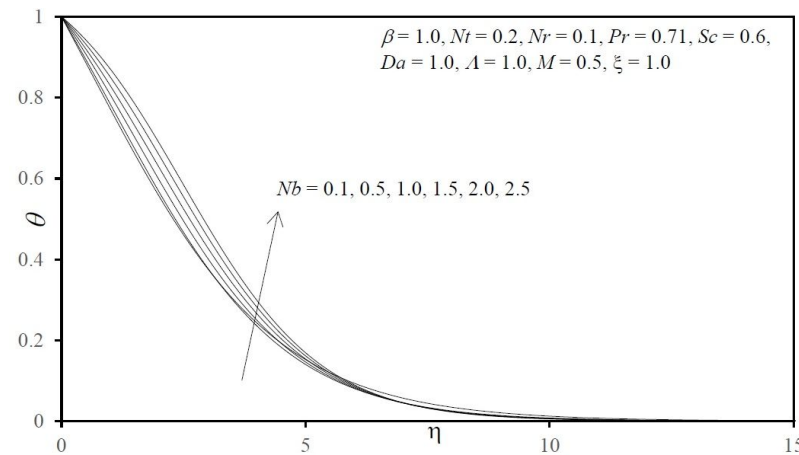

(b)

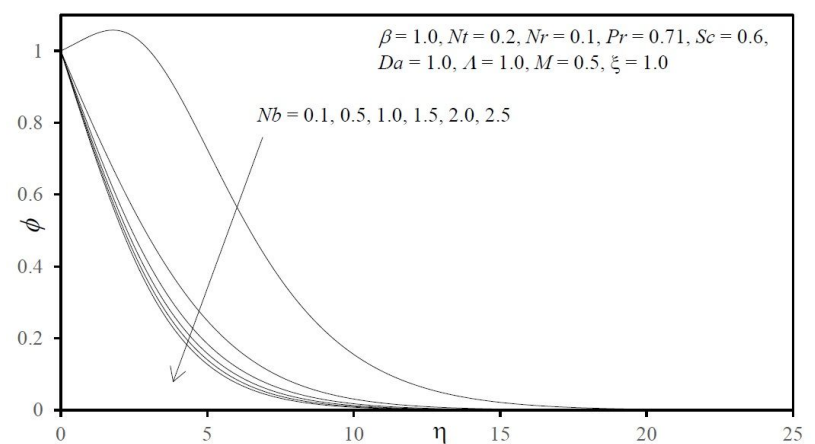

(c)

Fig. 4: (a) Influence of $N b$ on Velocity Profiles; (b) Influence of $N b$ on Temperature Profiles; (c) Influence of $\mathrm{Nb}$ on Concentration Profiles

terms and hence the effect of casson parameter is indirectly transmitted to both temperature and concentration filed. The similar trends are observed by Subba Rao et al. [60].

Figs. 4(a) - 4(c) presents the profiles of velocity $\left(f^{\prime}\right)$, temperature $(\theta)$ and concentration $(\varphi)$ for different values of $N b$. An increase in velocity is observed for various values of $\mathrm{Nb}$ throughout the boundary layer regime. Also, a slight increase in temperature is observed which is due to the collision between the random motions of the nanoparticles. Whereas, the species concentration is found to decrease with an increase in $\mathrm{Nb}$. Keblinski et al. [61] has elaborated

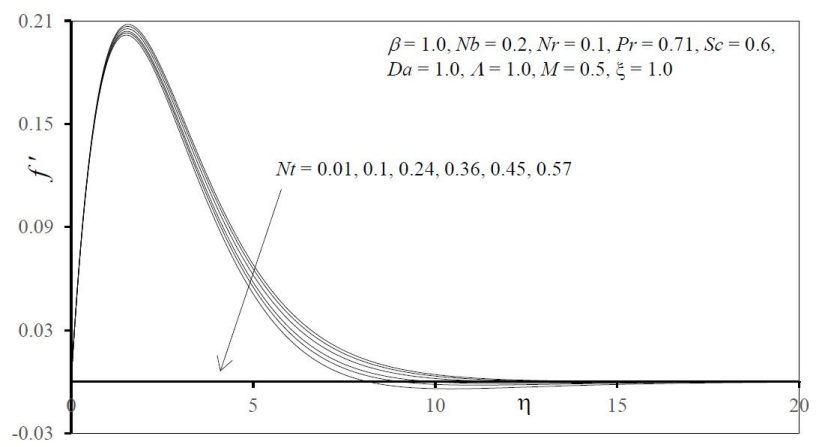

(a)

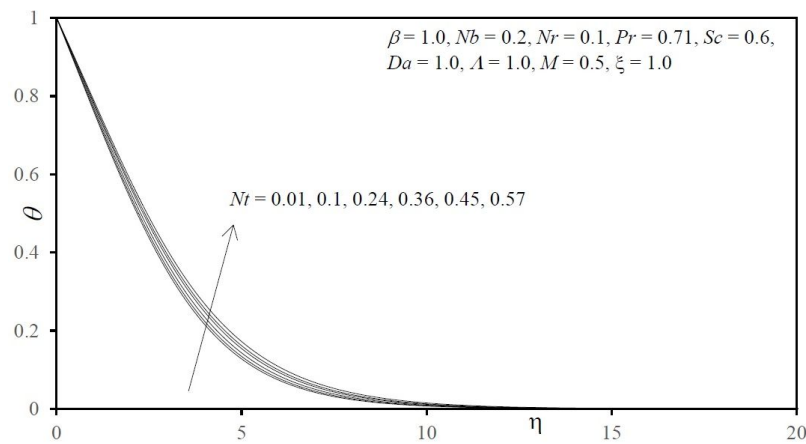

(b)

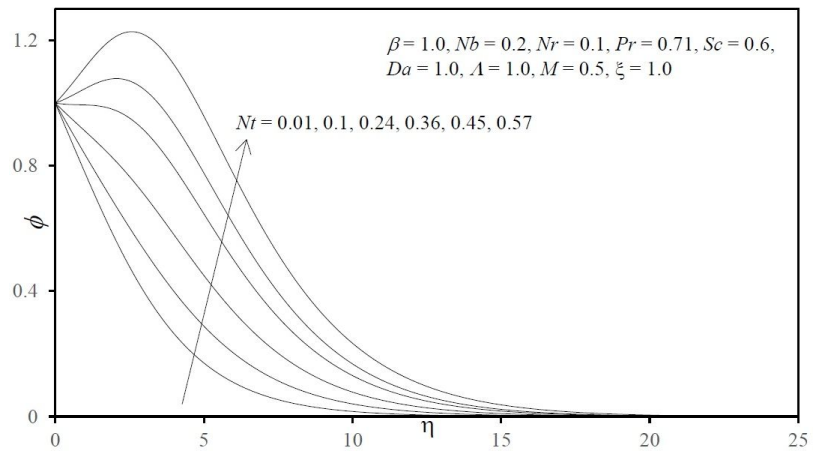

(c)

Fig. 5: (a) Influence of Nt on Velocity Profiles; (b) Influence of Nt on Temperature Profiles; (c) Influence of Nt on Concentration Profiles

various mechanisms which may contribute to enhancement in temperatures via augmentation of the thermal conductivity with nano-particles. These include Brownian motion of nanoparticles, ballistic transport of energy carriers within individual nanoparticles and between nanoparticles that are in contact, nanoparticle distribution and also the interfacial ordering of liquid molecules on the surface of nanoparticles. Brownian motion elevates thermal conduction via nanoparticles carrying thermal energy. However it has been seen that the direct contribution of Brownian motion is less significant as the time scale of the Brownian motion is about two orders of magnitude 


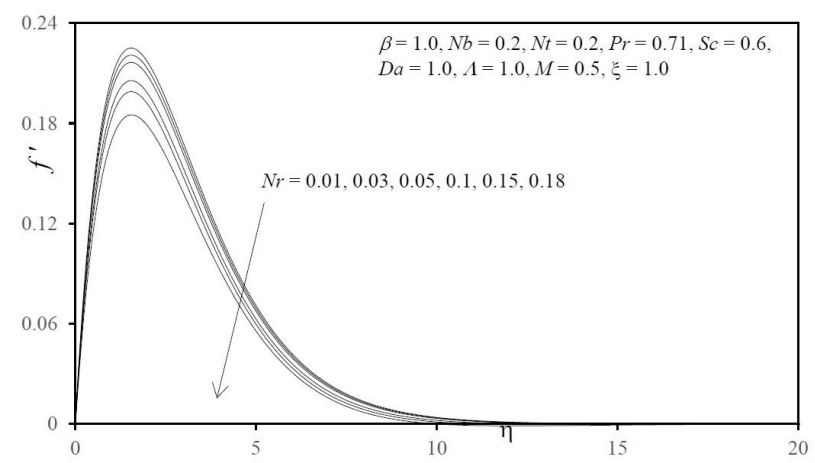

(a)

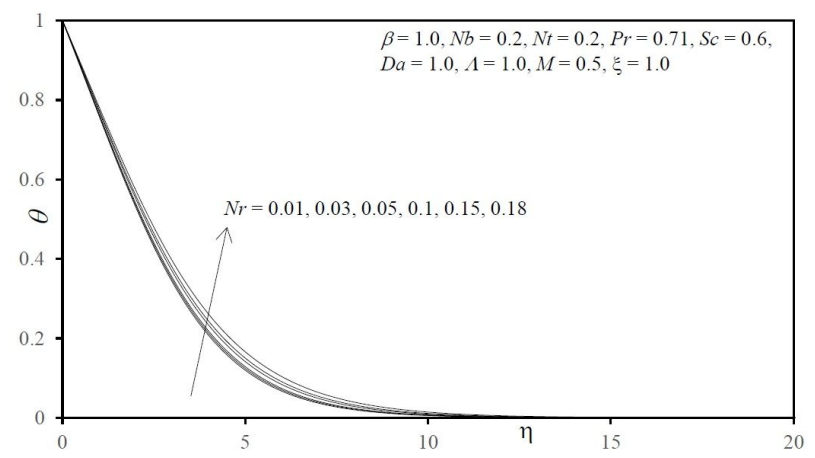

(b)

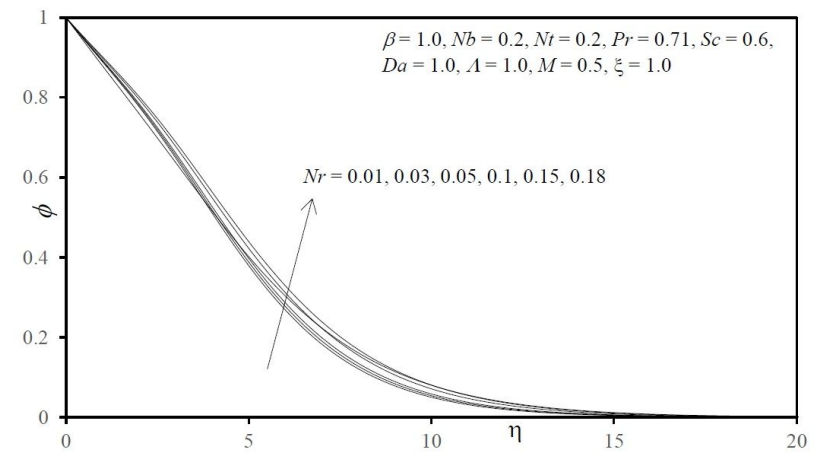

(c)

Fig. 6: (a) Influence of $\mathrm{Nr}$ on Velocity Profiles; (b) Influence of $\mathrm{Nr}$ on Temperature Profiles; (c) Influence of $\mathrm{Nr}$ on Concentration Profiles

larger than that for the thermal diffusion of the base liquid. Nanoparticles frequently are in the form of agglomerates and/or aggregates. For small particles, Brownian motion is strong and the parameter $N b$ will have high values; the opposite will apply for large particles.

Figs. 5(a) - 5(c) illustrates the impact of $N t$ on velocity $\left(f^{\prime}\right)$, temperature $(\theta)$ and concentration $(\varphi)$. A decrease in velocity is seen for various values of $N t$ throughout the boundary layer regime. A significant increase in both temperature and nano-particle concentration is generated as $N t$ increases. Thermophoresis heats the boundary layer and assists in particle deposition away from the

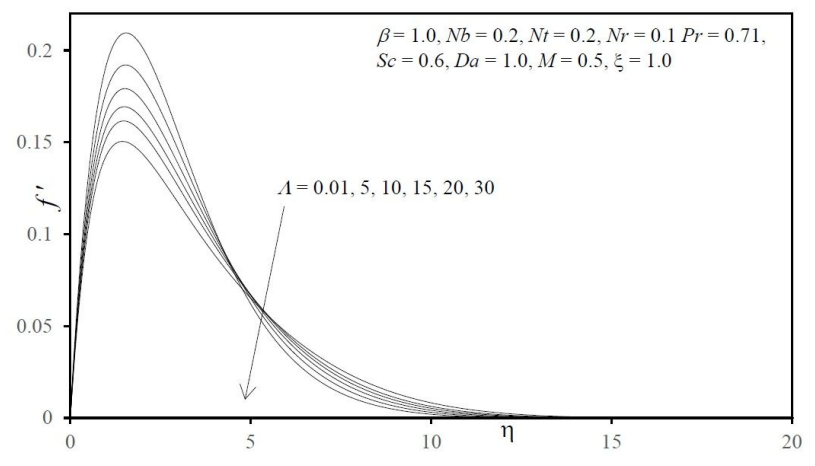

(a)

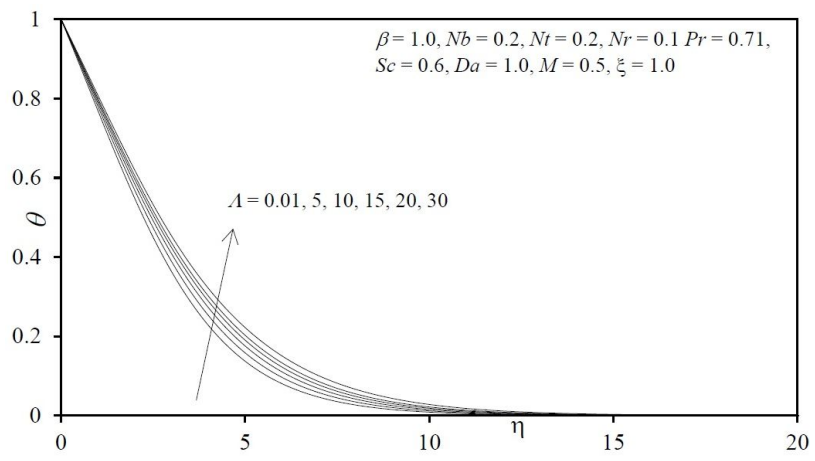

(b)

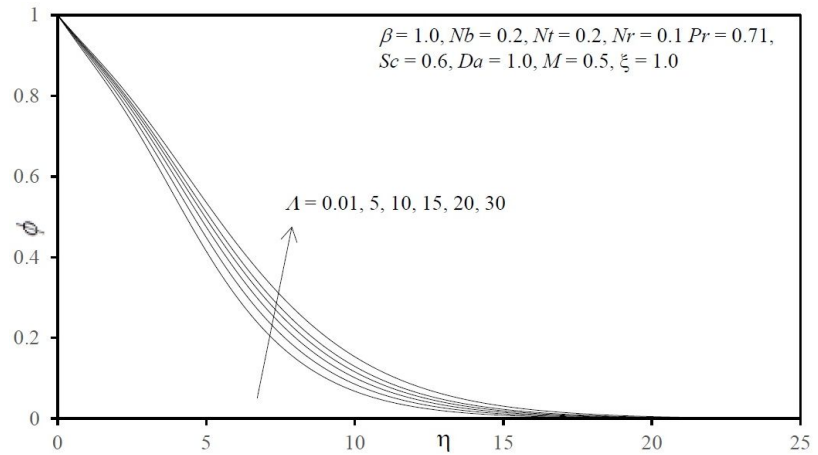

(c)

Fig. 7: (a) Influence of $\Lambda$ on Velocity Profiles; (b) Influence of $\Lambda$ on Temperature Profiles; (c) Influence of $\Lambda$ on Concentration Profiles

fluid regime, thereby accounting for the elevated concentration (nano-particle) as shown in Fig. 5(c). For $N t=0.1$, a monotonic decay in nano-particle concentration from the wall of the cylinder into the free stream. However, for $N t>$ 0.1 , a concentration peak is observed further from the surface of the cylinder.

Figs. 5(a) - 5(c) depicts the profiles for velocity $\left(f^{\prime}\right)$, temperature $(\theta)$ and concentration $(\varphi)$ for different values of $N r$. By definition, $\mathrm{Nr}$ represents the ratio of nanoparticle concentration to the thermal buoyancy force. The parameter $\mathrm{Nr}$, arises only in the momentum boundary layer equa- 


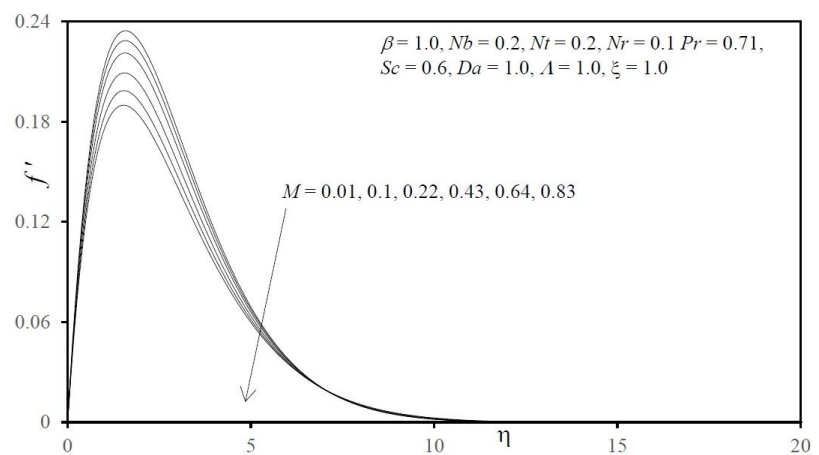

(a)

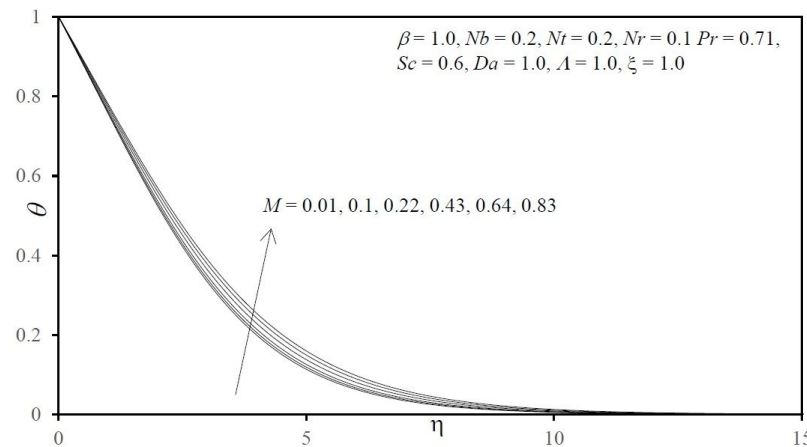

(b)

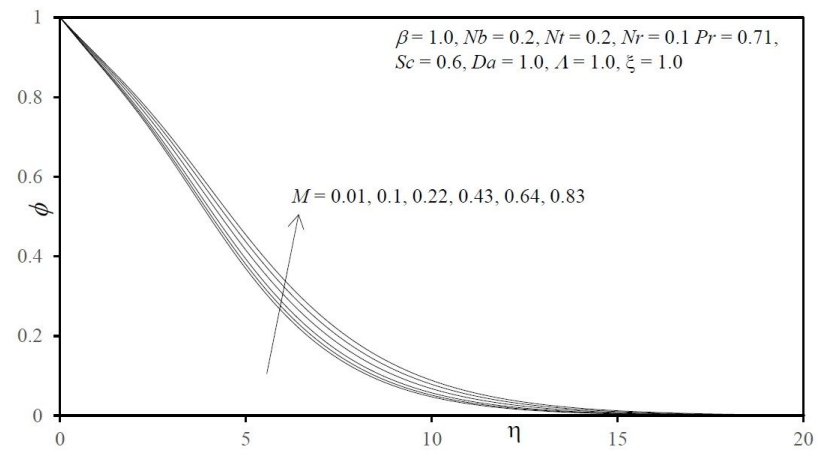

(c)

Fig. 8: (a) Influence of $M$ on Velocity Profiles; (b) Influence of $M$ on Temperature Profiles; (c) Influence of $M$ on Concentration Profiles

tion in the term $(\sin \xi / \xi)(\theta-N r \varphi)$ and couples the momentum equation to the thermal and concentration boundary layer equations respectively. With increasing $N r$ values, the velocity is decreased throughout the boundary layer. A slight increase in both temperature and concentration is observed. Hence, the buoyancy forces aid in the diffusion of heat and species (nano-particles) in the regime, whereas they induce a deceleration in the flow regime. Similar trends have been recently observed for other free convection nanofluid boundary layer flows by Gorla and Kumari [62].

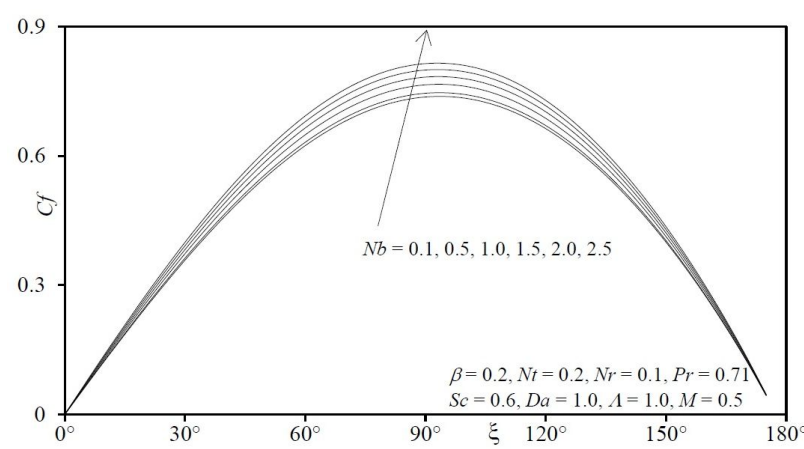

(a)

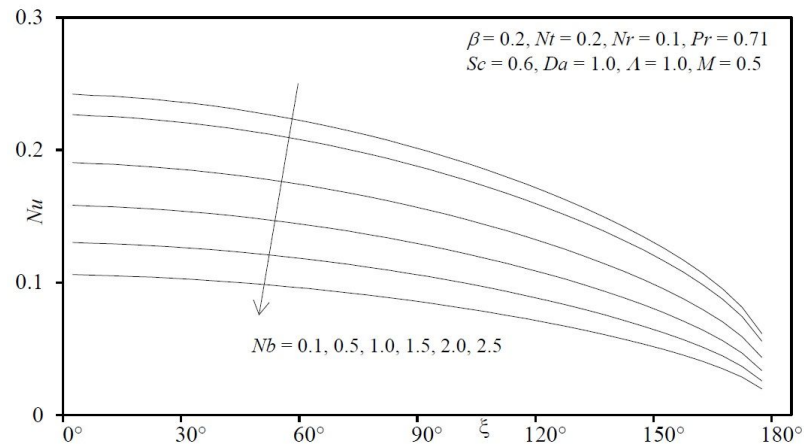

(b)

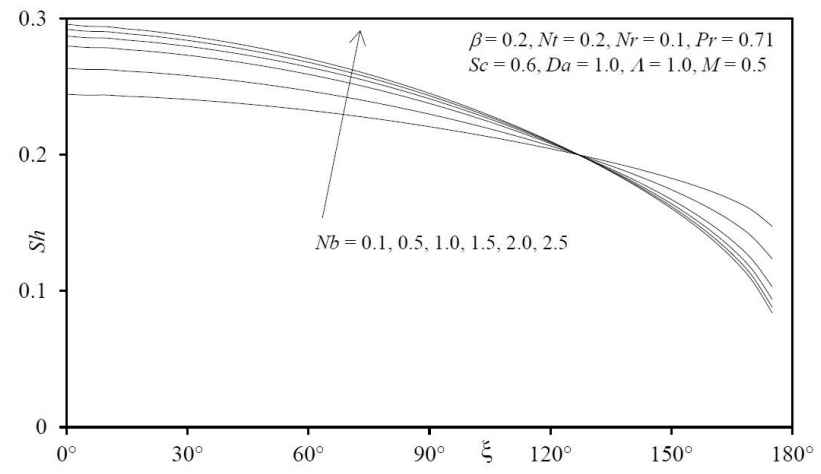

(c)

Fig. 9: (a) Influence of $\mathrm{Nb}$ on Skin Friction; (b) Influence of $\mathrm{Nb}$ on Nusselt Number; (c) Influence of $\mathrm{Nb}$ on Sherwood Number

Figs. 7(a) -7 (c) presents the impact of Forchheimer parameter, $\Lambda$ on velocity $\left(f^{\prime}\right)$, temperature $(\theta)$ and concentration $(\varphi)$. The parameter $\Lambda$ is associated with the second order Forchheimer resistance term, $-\xi \Lambda\left(f^{\prime}\right)^{2}$ in the momentum equation. The Forchheimer drag is directly proportional to $\Lambda$. As shown in Fig. 7(a), the flow is markedly decelerated with increasing values of $\Lambda$. As indicated by Kaviany [63], the Forchheimer effects are associated with higher velocities in porous media transport. A significant elevation in both temperature and nano-particel species diffusion concentration is seen with an increase in $\Lambda$. 


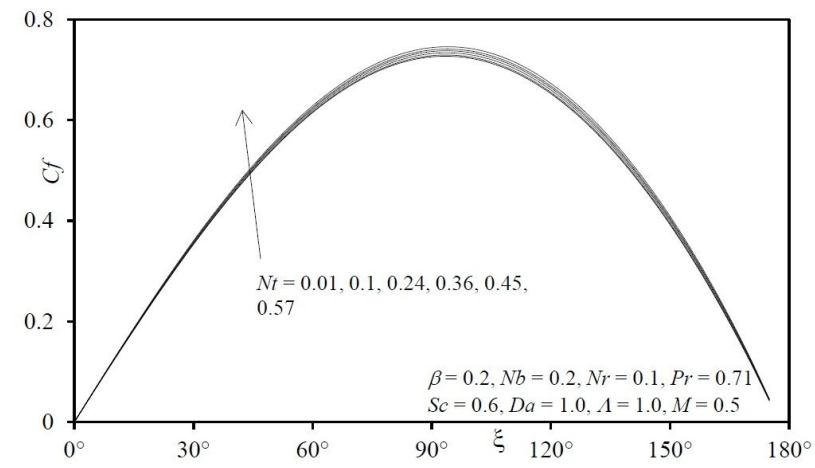

(a)

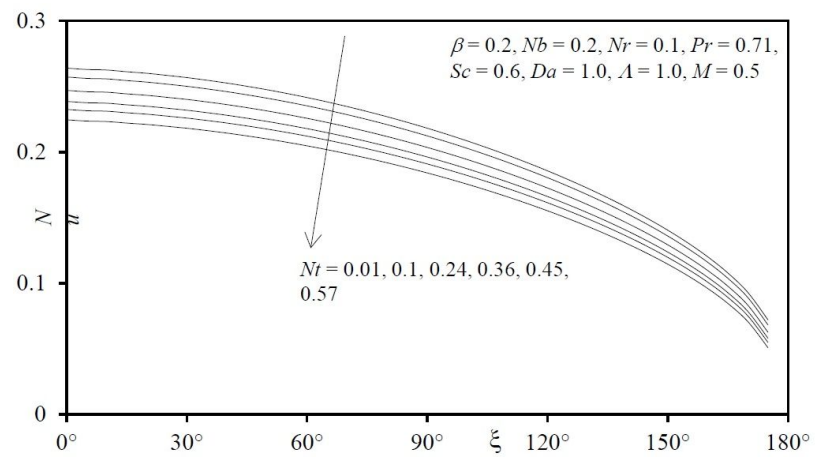

(b)

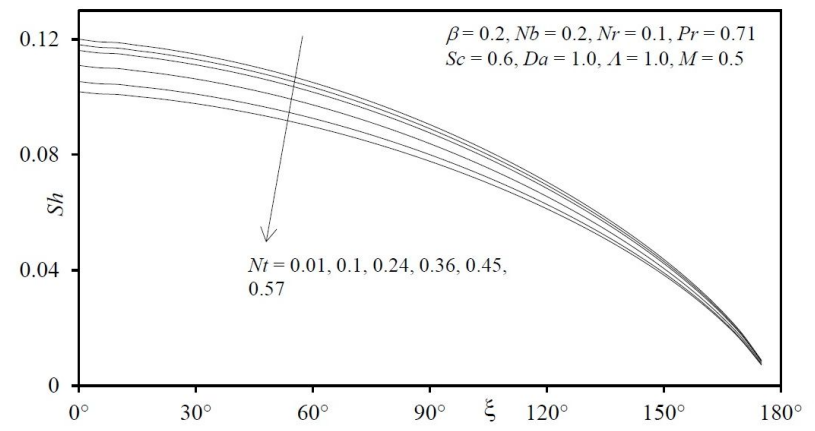

(c)

Fig. 10: (a) Influence of Nt on Skin Friction; (b) Influence of $N t$ on Nusselt Number; (c) Influence of $N t$ on Sherwood Number

Figs. 8(a) $-8(\mathrm{c})$ depict the profiles for velocity $\left(f^{\prime}\right)$, temperature $(\theta)$ and concentration $(\varphi)$ for increasing values of magnetic parameter, $M$. The parameter $M$ represents the ratio of magnetic Lorentzian drag force to viscous hydrodynamic force in the flow. For $M<1$, the viscous force dominates the magnetic force and the magnetohydrodynamic effect is weak. However, Fig. 8a shows that even a small increase in $M$ induces a marked deceleration in velocity. This reveals that the magnetic field resists the fluid transport due to rise in $\mathrm{M}$ lends an enhancement in the Lorentz force, which resist the fluid flow. This concurs with many other studies of magnetized nanofluid convec-

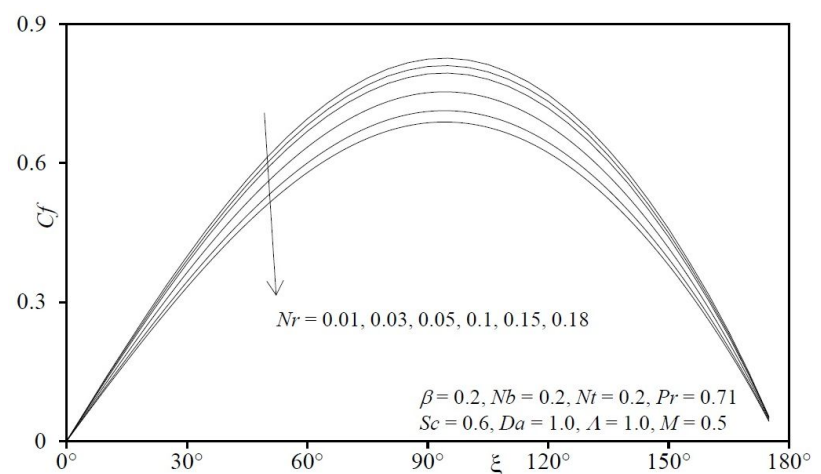

(a)

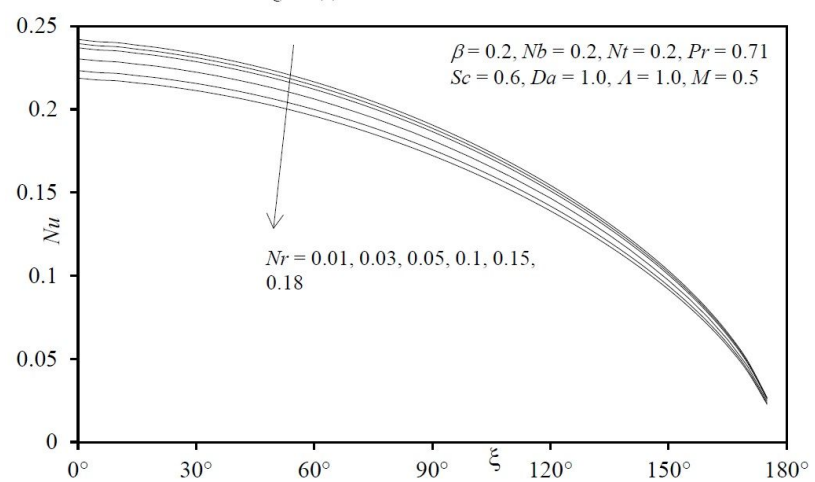

(b)

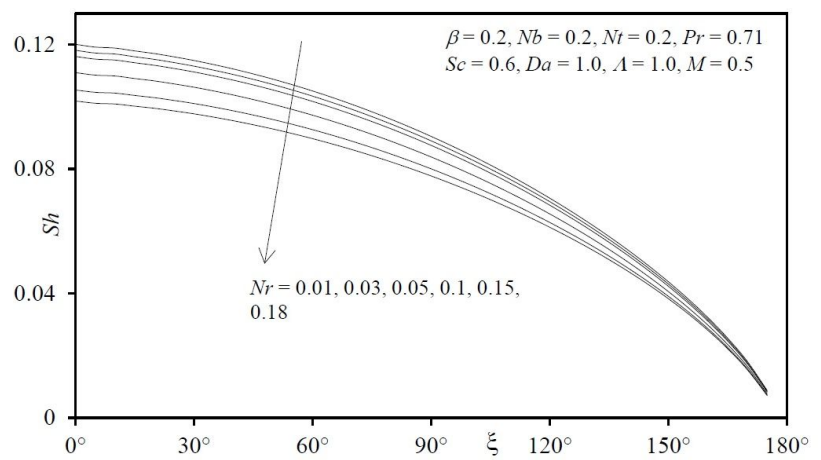

(c)

Fig. 11: (a) Influence of $\mathrm{Nr}$ on Skin Friction; (b) Influence of $\mathrm{Nr}$ on Nusselt; (c) Influence of $\mathrm{Nb}$ on Sherwood Number

tion since the radial magnetic field acts to generate a perpendicular drag force which acts to decelerate the flow. Figs. $8 \mathrm{~b}$ and $8 \mathrm{c}$ indicate that the dominant effect of greater magnetic parameter is to elevate both temperatures and nano-particle concentrations. The supplementary work expended in dragging the viscoplastic nanofluid against the action of the magnetic field manifests in kinetic energy dissipation. This energizes the boundary layer since the kinetic energy is dissipated as thermal energy and this further serves to agitate improved species diffusion. As a 


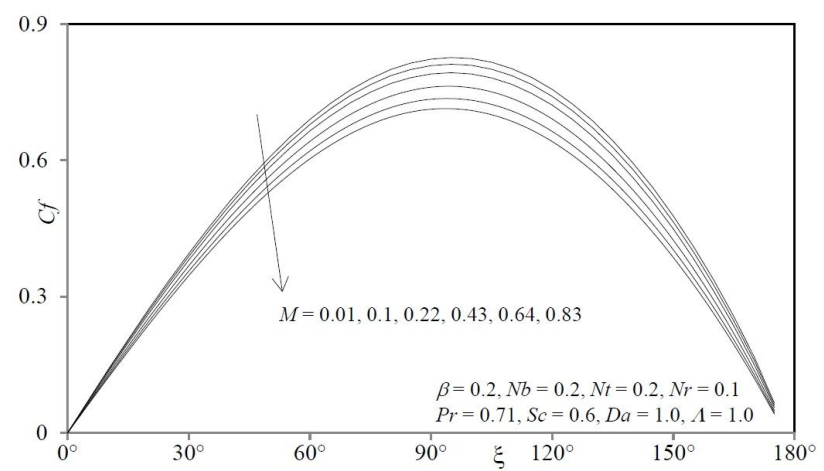

(a)

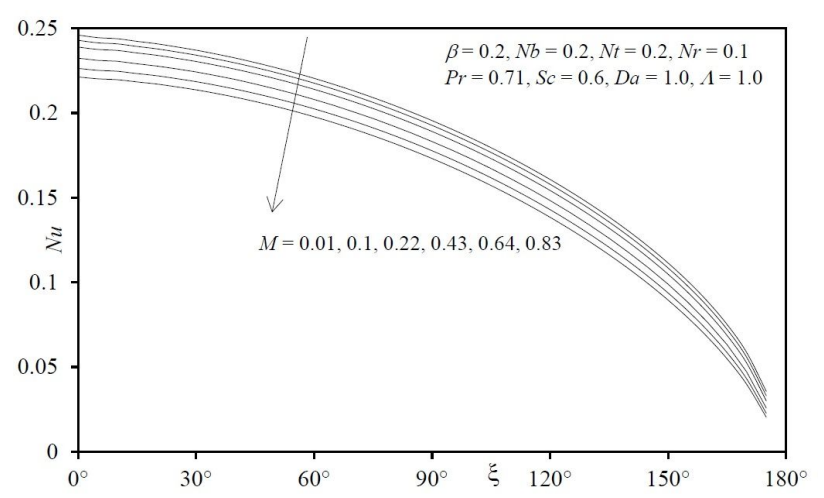

(b)

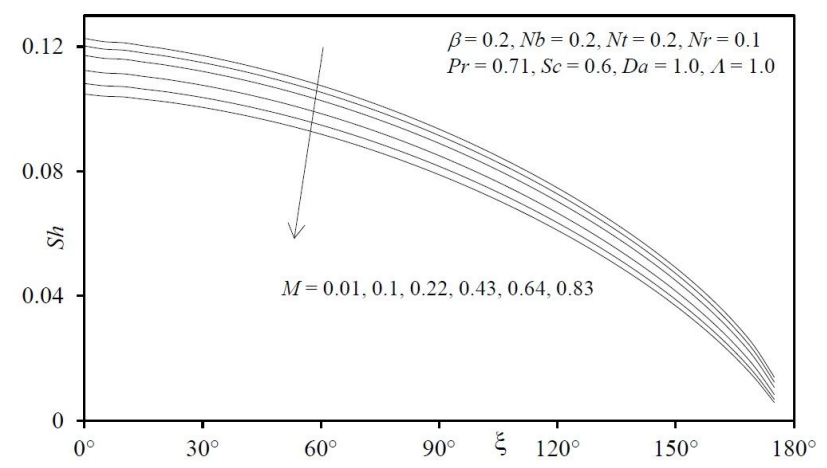

(c)

Fig. 12: (a) Influence of $M$ on Skin Friction; (b) Influence of $M$ on Nusselt Number; (c) Influence of $M$ on Sherwood Number

result both thermal and nano-particle (species) concentration boundary layer thicknesses are increased.

Figs. 9(a) - 9(c) presents the effects of $N b$ on skin friction $\left(C_{f}\right)$, heat transfer rate $(\mathrm{Nu})$ and mass transfer rate $(\mathrm{Sh})$ at the cylinder surface. $C_{f}$ is observed to increase slightly for different values of $\mathrm{Nb}$. Conversely, $\mathrm{Nu}$ is decreased significantly with increasing $N b$. There is also a progressive depletion in heat transfer rate with increasing transverse coordinate i.e. $x$-value. This agrees with the corresponding enhancement in temperature in the boundary layer owing to the nanofluid properties as represented by the Brownian diffusion effect. A decrease in heat transfer rate at the wall implies that less heat is convected from the fluid regime to the cylinder, thereby heating the boundary layer. Dimensionless mass transfer rate (local Sherwood number) is boosted up with increasing $N b$.

Figs. 10(a) - 10(c) depicts the profiles for on skin friction $\left(C_{f}\right)$, heat transfer rate $(\mathrm{Nu})$ and mass transfer rate $(\mathrm{Sh})$ at the cylinder surface for increasing $N t$ values. Clearly from Fig. 11(a) a very slight increase in $C_{f}$ is observed with an increase in $N t$ whereas, an increase in $N t$ decreases both heat transfer rate and mass transfer rate. Hence, thermophoresis exerts a significant effect on both heat and mass transfer characteristics at the cylinder surface.

Figs. 11(a) - 11(c) illustrate the responses of buoyancy ratio $\mathrm{Nr}$, on skin friction $\left(C_{f}\right)$, heat transfer rate $(\mathrm{Nu})$ and mass transfer rate $(S h)$ at the cylinder surface. For $\mathrm{Nr}>0$, the $C_{f}$ at the cylinder surface is observed to decrease for increasing values of $\mathrm{Nr}$. Also, the heat transfer rate and mass transfer rates are found to reduce with increasing $\mathrm{Nr}$ values.

Figs. 12(a) - 12(c) presents the responses of magnetic parameter $M$, on skin friction $\left(C_{f}\right)$, heat transfer rate $(\mathrm{Nu})$ and mass transfer rate $(S h)$ at the cylinder surface. A significant decrease in $C_{f}$ is observed at the cylinder surface for increasing values of $M$. Similar trends are observed in the case of heat and mass transfer rates i.e., both $\mathrm{Nu}$ and $\mathrm{Sh}$ are found to be strongly reduced with increasing $M$ values.

\section{Conclusions}

A numerical analysis is developed to study the laminar MHD convection flows of Casson nanofluid from an isothermal horizontal circular cylinder in fluid-saturated non-Darcy porous medium. An implicit finite difference Keller-Box technique is used to solve the non-dimensional boundary layer equations with prescribed boundary conditions. A comprehensive assessment of the impact of Casson fluid parameter $(\beta)$, Brownian motion parameter $(\mathrm{Nb})$, Thermophoresis parameter (Nt), Buoyancy ratio parameter (Nr), Darcy number (Da), Magnetic parameter (M), Forchheimer parameter ( $\Lambda$ ), Prandtl number (Pr) and Schmidt number (Sc). Very stable and accurate solutions are obtained with the present code. The present has been validated with the earlier Newtonian study. The present numerical code is able to solve the nonlinear rheological boundary layer flow problems very efficiently and hence presents excellent promise in simulating transport phenomena in other non-Newtonian fluids. 


\section{Nomenclature}

\begin{tabular}{|c|c|c|c|}
\hline$a$ & Radius of the cylinder & $u, v$ & $\begin{array}{l}\text { Dimensionless velocity components in } X \text { and } Y \text { direction } \\
\text { respectively }\end{array}$ \\
\hline$B_{0}$ & Constant imposed magnetic field & $x$ & Stream wise coordinate \\
\hline C & Fluid concentration & $y$ & Transverse coordinate \\
\hline$C f$ & Skin Friction Coefficient & \multicolumn{2}{|c|}{ Greek Symbols } \\
\hline$D a$ & Darcy Parameter & $\alpha$ & Thermal diffusivity \\
\hline$D_{B}$ & Brownian diffusion coefficient & $\beta$ & Casson fluid parameter \\
\hline$D m$ & Mass diffusion & $\beta_{1}$ & Coefficient of thermal expansion \\
\hline$D_{T}$ & Thermophoretic diffusion coefficient & $\Phi$ & Azimuthal coordinate \\
\hline$f$ & Dimensionless stream function & $\sigma$ & Electric conductivity of the fluid \\
\hline$g$ & Gravitational acceleration & $\eta$ & Non-dimensional radial coordinate \\
\hline$K$ & Thermal diffusivity & $\Gamma$ & Inertial drag coefficient \\
\hline$k$ & Thermal conductivity of the fluid & $\mu$ & Dynamic viscosity \\
\hline Gr & Grashof number & $\xi$ & Non-dimensional tangential coordinate \\
\hline M & Magnetic Parameter & $\psi$ & Non-dimensional stream function \\
\hline$N b$ & Brownian motion parameter & $v$ & Kinematic viscosity \\
\hline$N t$ & Thermophoresis parameter & $\varphi$ & Dimensionless concentration \\
\hline$N r$ & Buoyancy ratio parameter & $\theta$ & Dimensionless temperature \\
\hline $\mathrm{Nu}$ & Heat transfer coefficient & $\rho$ & Fluid density \\
\hline $\operatorname{Pr}$ & Prandtl number & $\Lambda$ & Forchheimer parameter (local inertial drag coefficient) \\
\hline$T$ & Fluid temperature & & \\
\hline$S$ & Cauchy stress tensor & \multicolumn{2}{|c|}{ Subscripts } \\
\hline Le & Lewis number & $w$ & Conditions on the wall \\
\hline Sh & Sherwood number & $\infty$ & Free stream condition \\
\hline
\end{tabular}

\section{References}

[1] Schaefer HE., Nanoscience. The science of the small in physics, engineering, chemistry, biology and medicine, Heidelberg: Springer; 2010. DOI: 10.1007/978-3-642-10559-3.

[2] Kleinstrauer C., Microfluidics and Nanofluidics, Theory and selected applications, New Jersey: Wiley; 2013.

[3] Choi S., Enhancing thermal conductivity of fluids with nanoparticles". ASME-Publ. Fluids Engineering Division, 1995, 231, 99-106.

[4] Buongiorno J., Convective transport in Nanofluids, ASME J Heat Trans., 2006, 128(3), 240-250. Doi:10.1115/1.2150834.

[5] M. Sheikholeslami, Milad Darzi, M.K. Sodaughi, Heat transfer improvement and pressure drop during condensation of refrigerant-based nanofluid; an experimental procedure, Int. J. Heat and Mass Trans., 2018, 122, 643-650.

[6] M. Sheikholeslami, Houman B. Rokni, CVFEM for effect of Lorentz forces on nanofluid flow in a porous complex shaped enclosure by means of non-equilibrium model, J. Molecular Liquids, 2018, 254, 446-462.

[7] K. M. Shirvan, R. Ellahi, Mojtaba Mamourian, Mohammad Mamourian, Effect of Wavy Surface Characteristics on natural convection heat transfer in a cosine corrugated square cavity filled with Nanofluid, Int. J. Heat and Mass Trans., 2017, 107, 1110-1118.

[8] Saman Rashidi, Shima Akar, Masoud Bovand, Rahmat Ellahi, Volume of fluid model to simulate the nanofluid flow and entropy generation in a single slope solar still, Renewable Energy, 2018, 115, 400-410.
[9] Sheikholeslami M. and S.A. Shehzad, Simulation of water based nanofluid convective flow inside a porous enclosure via non-equilibrium model, Int. J. Heat and Mass Trans., 2018, 120, 1200-1212.

[10] Sheikholeslami, Numerical investigation of nanofluid free convection under the influence of electric filed in a porous enclosure, J. Molecular Liquids, 2018, 249, 1212-1221.

[11] K. M. Shirvan, Mojtaba Mamourian, Soroush Mirzakhanlari, R. Ellahi, Numerical Investigation of Heat Exchanger Effectiveness in a Double Pipe Heat Exchanger Filled With Nanofluid: A Sensitivity Analysis by Response Surface Methodology, Power Techn., 2017, 313, 99-111.

[12] J. A. Esfahani, M. Akbarzadeh, S. Rashidi, M.A. Rosen, R. Ellahi, Influences of wavy wall and nanoparticles on entropy generation over heat exchanger plat, Int. J. Heat and Mass Trans., 2017, 109, 1162-1171.

[13] Nasir Ali, Hayat T., Saleem Asghar, Peristaltic Flow of a Maxwell fluid in a channel with compliant walls, Chaos Solitons \& Fractals, 2009, 39(1), 407-416. DOI:10.1016/j.chaos.2007.04.010.

[14] Hayat T., Qasim Muhammad, Abbas Zaheer, Hendi Awatif A., Magnetohydrodynamic flow and mass Transfer of a Jeffrey Fluid over a Nonlinear Stretching surface, Zeitschrift für Naturforschung A, 2014, 65(12), 1111-1120. DOI:10.1515/zna-20101216.

[15] T. F. Irvine, J. Karni, Non-Newtonian fluid flow and heat transfer, Handbook of Single-Phase Convective heat transfer, Chapter 20, Wiley New York, 1987, 20.1-20.57.

[16] Y. Y. Lok, I. Pop, D. B. Ingham, Oblique stagnation slip flow of a micropolar fluid, Meccanica, 2010, 45(2), 187-198. 
[17] Azad Hussain, Anwar Ullah, Boundary layer flow of a Walter's B fluid due to a stretching cylinder with temperature dependent viscosity, Alex. Eng. J., 2016, 55(4), 3073-3080.

[18] S. Abdul Gaffar, V. Ramachandra Prasad, E. Keshava Reddy, Computational study of Jeffrey's non-Newtonian fluid past a semi-infinite vertical plate with thermal radiation and heat generation/absorption, Ain Shams Eng. J., 2017, 8(2), 277-294.

[19] A. Subba Rao, C.H. Amanulla, N. Nagendra, O. Anwar Beg, A. Kadir, Hydromagnetic flow and heat transfer in a Williamson Non-Newtonian fluid from a Horizontal circular cylinder with Newtonian Heating, Int. J. Appl. Comp.. Math., 2017, 3(4), 3389-3409. DOI: 10.1007/s40819-017-0304-x

[20] Ramachandra Prasad V., S. Abdul gaffar and O. Anwar Bég, Non-Similar computational solutions for free convection boundary layer flow of a nanofluid from an isothermal sphere in a Non-Darcy porous medium, J. Nanofluids, 2015, 4(2), 203213.

[21] Huicui Li, Yongjun Jian, Dispersion for periodic electro-osmotic flow of Maxwell fluid through a microtube, Int. J. Heat and Mass Trans., 2017, 115A, 703-713.

[22] S. Abdul Gaffar, V. Ramachandra Prasad, O. Anwar Beg, Computational study of non-Newtonian Eyring-Powell fluid from a vertical porous plate with Biot number effects, J. Braz. Soc. Mech. Sci. Eng., 2017, 39(7), 2747-2765.

[23] S. Abdul Gaffar, V. Ramachandra Prasad, E. Keshava Reddy, Computational Study of MHD free convection flow of nonNewtonian Tangent Hyperbolic fluid from a vertical surface in porous media with Hall/lonslip current and Ohmic dissipation, Int. J. Appl. Comp. Math, 2017, 3(2), 859-890.

[24] R. Mehmood, S. Rana, S. Nadeem, Transverse thermophoretic MHD Oldroyd-B fluid with Newtonian heating, Results in Physics, 2018, 8, 686-693.

[25] N. ljaz, A. Zeeshan, M. M. Bhatti, R. Ellahi, Analytical study on liquid-solid particles interaction in the presence of heat and mass transfer through a wavy channel, J. Molecular Liquids, Vol. 250, 2018, 80-87.

[26] Casson, N., A Flow Equation for Pigment Oil-Suspensions of the Printing Ink Type, in: C.C. Mill Ed., Rheology of Disperse Systems, London: Pergamon Press, 1959, 84-102.

[27] Bird, R.B., Dai, G.C., and Yarusso, B.J., The Rheology and Flow of Viscoplastic Materials, Rev. Chem. Eng, 1983, 1, 1-83.

[28] G. Kumaran, N. Sandeep, Thermophoresis and Brownian moment effects on parabolic flow of MHD Casson and Williamson fluids with cross diffusion, J. Molecular Liquids, 2017, 233, 262-269. doi: 10.1016/j.molliq.2017.03.031.

[29] M.I. Khan, M. Waqas, T. Hayat, A. Alsaedi, Colloidal study of Casson fluid with homogeneous-heterogeneous reactions, J. Colloid and Interface Science, 2017, 498, 89-90. http://dx.doi.org/10.1016/j.jcis.2017.03.024

[30] M. Nawaz, Rahila Naz, M. Awais, Magnetohydrodynamics Axisymmetric flow of Casson fluid with variable thermal conductivity and free stream, Alex. Eng. J., 2017. http://dx.doi.org/10.1016/j.aej.2017.05.016

[31] Kashif Ali Khan, Asma Rashid Butt, Nauman Raza, Effects of heat and mass transfer on unsteady boundary layer flow of a chemical reacting Casson fluid, Results in Physics, 2018, 8, 610-620. https://doi.org/10.1016/j.rinp.2017.12.080

[32] Sameh E. Ahmed, M.A. Mansour, A. Mahdy, Shadia S. Mohamed, Entropy generation due to double diffusive convective flow of Casson fluids over nonlinearity stretching sheets with slip conditions, Eng. Sci. Tech., an Int. J., Vol. 20, Issue 6, pp.1553-1562, 2017.

[33] B. Mahanthesh, B. J, Gireesh, Scrutinization of thermal radiation, viscous dissipation and Joule heating effects on Marangoni convective two-phase flow of Casson fluid with fluid-particle suspension, Results in Physics, 2018, 8, 869878.

[34] Khalil Ur Rehman, Aqeela Qaiser, M.Y. Malik, U. Ali , Numerical communication for MHD thermally stratified dual convection flow of Casson fluid yields by stretching cylinder, Chinese Journal of Physics, 2017, 55(4), doi: 10.1016/j.cjph.2017.05.002.

[35] A. Kumar and A. K. Singh, Effect of induced magnetic field on natural convection in vertical concentric annuli heated/cooled asymmetrically, J. Appl. Fluid Mech., 2013, 6(1), 15-26.

[36] Kartini Ahmad, Anuar Ishak, Magnetohydrodynamic (MHD) Jeffrey fluid over a stretching vertical surface in a porous medium, Propulsion and Power Research, 2017, 6(4), 269-276. http://dx.doi.org/10.1016/j.jppr.2017.11.007

[37] Sheikholeslami and Houman B. Rokni, Magnetic nanofluid flow and convective heat transfer in a porous cavity considering Brownian motion effects, Physics of Fluids, 2018, 30(1). https://doi.org/10.1063/1.5012517.

[38] Sheikholeslami, CuO-water nanofluid flow due to magnetic field inside a porous media considering Browinian motion, $J$. Mole. Liquids, 2018, 249, 921-929.

[39] Mohsan Hassan, Ahmad Zeeshan, Aaqib Majeed, Rahmat Ellahi, Particle shape effects on ferrofuids flow and heat transfer under influence of low oscillating magnetic field, J. Magnetism and Magnetic Materials, 2017, 443, 36-44.

[40] R. Ellahi, M.H. Tariq, M. Hassan, K. Vafai, On boundary layer nano-Ferroliquid flow under the influence of low oscillating stretchable rotating disk, J. Mole. Liquids, 2017, 229, 339-345.

[41] Zhi-Yong Xie, Yong-Jun Jian, Entropy generation of two-layer magnetohydrodynamic electoosmotic flow through microparallel channels, Energy, 2017. doi: 10.1016/j.energy.2017.08.038.

[42] A. Zeeshan, N. Shehzad, R. Ellahi, Ananlysis of activation energy in Couette-Poiseuille flow of nanofluid in the presence of chemical reaction and convective boundary conditions, Results in Physics, 2018, 8, 502-512.

[43] S. Abdul Gaffar, V. Ramachandra Prasad, E. Keshava Reddy, Magnetohydrodynamics flow of Non-Newtonian fluid from a vertical permeable cone in the presence of thermal radiation and heat generation/absorption, Int. J. Appl. Comput. Math., 2017, 3(4), 2849-2872.

[44] S. Abdul Gaffar, V. Ramachandra Prasad, E. Keshava Reddy, Magnetohydrodynamic free Convection flow and heat transfer of non-Newtonian Tangent Hyperbolic fluid from horizontal circular cylinder with Biot number effects, Int. J. Appl. Comput. Math., 2017, 3(2), 721-743.

[45] S. Abdul Gaffar, V. Ramachandra Prasad, E. Keshava Reddy, 0. Anwar Beg, Magnetohydrodynamic free convection boundary layer flow of non-Newtonian Tangent Hyperbolic fluid from a vertical permeable cone with variable surface temperature, $J$. Braz. Soc. Mech. Sci. Eng., 2017, 39(1), 101-116.

[46] O. Anwar Beg, S. Abdul gaffar, V. Ramachandra Prasad, M.J. Uddin, Computational solutions for non-isothermal, nonlinear magneto-convection in porous media with hall/ionslip currents and ohmic dissipation, Eng. Sci. Tech., an Int. J., 2016, 
19(1), 377-394.

[47] Bear, J., Dynamics of Fluids in Porous Media, Dover, New York 1988.

[48] D. Srinivasacharya, Ch. RamReddy, P. Naveen, O. Surender, Non-Darcy Mixed convection flow past a vertical porous media plate with Joule Heal, Hall and Ion-Slip Effects, Procedia Engineering, 2015, 127, 162-169.

[49] B. J. Gireesh, B. Mahanthesh, P. T. Manjunatha, R.S.R. Gorla, Numerical solution for hydromagnetic boundary layer flwo and heat transfer past a stretching surface embedded in non-Darcy porous medium with fluid-particle suspension, J. Nigerian Mathematical Society, 2015 34(3), 267-285. https://doi.org/10.1016/j.jnnms.2015.07.003

[50] Natalia c. Rosca, Alin V. Roşca, Teodor Groşan, Ioan Pop, Mixed convection boundary layer flow past a vertical flat plate embedded in a non-Darcy porous medium saturated by a nanofluid, Int. J. Numerical Methods for Heat \& Fluid Flow, 2014, 24(5), 970-987. https://doi.org/10.1108/HFF-09-20120199

[51] Hady, F. M.; Mohamed, R. A.; Mahdy, A.; Abo Zaid, Omima A., Non-Darcy natural convection boundary layer flow over a vertical cone in porous media saturated with a nanofluid containing Gyrotactic microorganisms with a convective boundary condition, J. Nanofluids, 2016, 5(5), 765-773. DOI: https://doi.org/10.1166/jon.2016.1256

[52] V. Ramachandra Prasad, S. Abdul Gaffar, O. Anwar Beg, Nonsimilar computational solutions for free convection boundarylayer flow of a Nanofluid from an isothermal sphere in a nonDarcy porous medium, J Nanofluids, 2015, 4(2), 1-11.

[53] Som, N. M., Arifin, N. M., Ali, F. M., \& Nazar, R., Boundary layer flow over a permeable stretching sheet embedded in a NonDarcian porous medium with thermal radiation and ohmic dissipation. Int. J. Math. Models and Methods in Appl. Sci., 2014, 8(1), 91-94.
[54] Ahmad Nazri Mohd Som, Norihan Md Arifin, Fadzilah Md Ali, Norfifah Bachok, Roslinda Nazar, Slip Effects on MHD NonDarcy Boundary layer Flow over a Stretching Sheet in a Porous Medium with radiation and Ohmic Dissipation. Int. Theor. Appl. Mech., 2016, 1, 13-18.

[55] S. Abdul Gaffar, V. Ramachandra Prasad E. Keshava Reddy, MHD free convection flow of non-Newtonian Eyring-Powell fluid from vertical surface in porous media with Hall/Ionslip currents and Ohmic dissipation, Alex. Eng. J., 2016, 55, 875905.

[56] S. Abdul Gaffar, V. Ramachandra Prasad, E. Keshava Reddy, 0. Anwar Beg, Numerical study of non-Newtonian Jeffrey's fluid from a permeable horizontal isothermal cylinder in non-Darcy porous medium, J. Braz. Soc. Mech. Sci. Eng., 2015, 37(6), 1765-1783.

[57] Keller, H.B., A new difference method for parabolic problems, J. Bramble (Editor), Numerical Methods for Partial Differential Equations, Academic Press, New York, USA, 1970.

[58] J.H. Merkin, Free convection boundary layers on cylinders of elliptic cross section, J. Heat Transfer, 1977, 99, 453-457.

[59] K.A. Yih, Effect of blowing/suction on MHD-natural convection over horizontal cylinder: UWT or UHF, Acta Mech., 2000, 144, 17-27.

[60] A. Subba Rao, V.R. Prasad, V. Naga Radhika, O. Anwar Beg, Heat Transfer in viscoplastic boundary-layer flow from a vertical permeable cone with momentum and thermal wall slip: numerical study, Heat Transfer Research, 2018, 49(1), 1-16. DOI:10.1615/HeatTransRes.2017018153

[61] Keblinski, P., Phillpot S.R., Choi S.U.S. and Eastman J.A., Mechanisms of heat flow in suspensions of nanosized particles (nanofluids), Int. J. Heat Mass Trans., 2002, 45, 855-863.

[62] Gorla, R.S.R. and M. Kumari, Free convection along a vertical wavy surface in a nanofluid Proc. ImechE- Part N: J. Nanoengineering and Nanosystems, 2011, 225, 133-142.

[63] Kaviany, M., Principles of Heat Transfer in Porous Media, MacGraw-Hill, New York 1992. 